\title{
Aportaciones a la flora de Galicia, $X$
}

\author{
Rubén Pino Pérez ${ }^{1}$, Francisco Javier Silva-Pando ${ }^{1,2}$, Antonio Galán de Mera ${ }^{3}$ Xosé Ramón García Martínez, \\ Juan José Pino Pérez ${ }^{4}$, María José Rozados Lorenzo ${ }^{1}$, Sandra González Pazos ${ }^{1}$, Fermín Gómez Vigide \\ José Luis Camaño Portela ${ }^{6}$, Simón Rial Pousa ${ }^{1}$, David Álvarez Graña ${ }^{1}$ y Jaime B. Blanco Dios ${ }^{1}$
}

Dedicamos este trabajo a nuestros queridos colegas y amigos Enrique Valdés Bermejo, Francisco Pérez Caramés, J. J. Moldes Ferro, Luis Freire y Santiago Castroviejo, tristemente desaparecidos, que nos han acompañado en más de una ocasión, y con su trabajo, esfuerzo y dedicación, hemos podido llegar hasta aquí.

Resumen: Pino Pérez, R.; Silva-Pando, F. J.; Galán de Mera, A.; García Martínez, X. R.; Pino Pérez, J. J.; Rozados Lorenzo, M. J.; González Pazos, S.; Gómez Vigide, F.; Camaño Portela, J. L.; Rial Pousa, S.; Álvarez Graña, D. \& Blanco Dios, J. B. 2011. Aportaciones a la flora de Galicia, X. 2011. Bot. Complut. 35: 65-87.

En este trabajo se mencionan 79 plantas de diverso interés para la flora de Galicia y otras zonas de la Península ibérica. Se incluyen 1 novedades para España (Watsonia bulbillifera), 24 novedades regionales (Crassula arborescens, Teline monspessulana, Hippocrepis comosa, Chamaesyce polygonifolia, Myosotis congesta, Verbena bonariensis, Senecio pyrenaicus, Taraxacum acutangulum, T. braun-blanquetii, T. cantabricum, T. ekmanii, T. fulgidum, T. hispanicum, T. lambinonii, T. maculosum, T. pinto-silvae, T. rubicundum, T. sundbergii, Agropyrum cristatum subsp. pectinatum, Bromus inermis, Pennisetum clandestinum, Eragrostis mexicana var. virescens, Iris planifolia, Chasmanthe floribunda), 26 novedades provinciales (Ranunculus peltatus subsp. peltatus var. peltatus, Modiola caroliniana, Sedum dendroideum, Paraserianthes lophantha, Geranium purpureum, Calystegia silvatica subsp. disjuncta, Echium vulgare subsp. pustulatum, Linaria aguillonensis, Linaria lamarckii, Glechoma hederacea, Asperula aristata subsp. scabra, Taraxacum drucei, T. gallaecicum, T. marklundii, T. nordstedtii, Luzula lactea, Commelina communis, Canna indica, Cyperus involucratus, Paspalum notatum, Asphodelus fistulosus, Muscari neglectum, Veratrum album, Iris foetidissima y Hedychium gardnerianum, Passiflora caerulea) y otras 15 de interés diverso, bien ecológico, corológico o taxonómico. Se confirma la presencia de Clypeola jonthlaspi, Alchemilla transiens, Asperula cynanchica subsp. cynanchica, Valeriana tripteris subsp. tripteris, Luzula sudetica y Rhynchospora modesti-lucennoi. También, y a la luz de la información obtenida, eliminamos del catálogo de la flora vascular de Galicia 6 taxones (Iberis amara, I. linifolia, Sedum sediforme, Anarrhinum laxiflorum, Asperula aristata subsp. aristata y Taraxacum palustre) y Carex vesicaria del Parque Nacional de las Illas Atlánticas.

Palabras clave: flora vascular, corología, ecología, Galicia, España.

Abstract: Pino Pérez, R.; Silva-Pando, F. J.; Galán de Mera, A.; García Martínez, X. R.; Pino Pérez, J. J.; Rozados Lorenzo, M. J.; González Pazos, S.; Gómez Vigide, F.; Camaño Portela, J. L.; Rial Pousa, S.; Álvarez Graña, D. \& Blanco Dios, J. B. 2011. Contributions to the flore of Galicia, X. 2011. Bot. Complut. 35: 65-87.

In this study mentioned 79 different plants with interest to the flora of Galicia and other territories of the Iberian Peninsula. One of them are new records for Spain flora (Watsonia bulbillifera), 24 are regional novelties (Crassula arborescens, Teline monspessulana, Hippocrepis comosa, Chamaesyce polygonifolia, Myosotis congesta, Verbena bonariensis, Senecio pyrenaicus, Taraxacum acutangulum, T. braun-blanquetii, T. cantabricum, T. ekmanii, T. fulgidum, T. hispanicum, T. lambinonii, T. maculosum, T. pinto-silvae, T. rubicundum, T. sundbergii, Agropyrum cristatum subsp. pectinatum, Bromus inermis, Pennisetum clandestinum, Eragrostis mexicana var. virescens, Iris planifolia, Chasmanthe floribunda), 26 novedades provinciales (Ranunculus peltatus subsp. peltatus var. peltatus, Modiola caroliniana, Sedum dendroideum, Paraserianthes lophantha, Geranium purpureum, Calystegia silvatica subsp. disjuncta, Echium vulgare subsp. pustulatum, Linaria aguillonensis, Linaria lamarckii, Glechoma hederacea, Asperula aristata subsp. scabra, Taraxacum drucei, T. gallaecicum, T. marklundii, T. nordstedtii, Luzula lactea, Commelina communis, Canna indica, Cyperus involucratus, Paspalum notatum, Asphodelus fistulosus, Muscari neglectum, Veratrum album, Iris foetidissi$m a$ and Hedychium gardnerianum, Passiflora caerulea). Also 15 of them are commented by its taxonomic, ecologic or chorological interest. Clypeola jonthlaspi, Alchemilla transiens, Asperula cynanchica subsp. cynanchica, Valeriana tripteris subsp. tripteris, Luzula sudetica and Rhynchos-

\footnotetext{
${ }^{1}$ Centro de Investigación Forestal de Lourizán, Dirección Xeral de Montes, Xunta de Galicia, Apartado 127, E-36080 Pontevedra, España. francisco.javier.silva.pando@xunta.es

${ }^{2}$ Departamento de Producción Vegetal, Escola Politécnica Superior, Universidade de Santiago de Compostela, Aguas Férreas s/n, Lugo, España.

${ }^{3}$ Laboratorio de Botánica, Facultad de Farmacia, Universidad San Pablo-CEU, Boadilla del Monte, E-28660 Madrid, España.

${ }^{4}$ Facultad de Ciencias, Universidad de Vigo, Lagoas-Marcosende s/n, E-36310 Vigo, España

${ }^{5}$ PP Franciscanos, E-15291 Louro, Muros, A Coruña, España.

${ }^{6}$ ETS Ingenieros Industriales, Universidad de Vido, Lagoas-Marcosende s/n, E-36310 Vigo, España

Recibido: 18 mayo 2011. Aceptado: 16 junio 2011
} 
pora modesti-lucennoi are confirmed for the Galician flora. Morever, in the light of further information, we remove 6 plants from the catalog of the vascular flora of Galicia (Iberis amara, I. linifolia, Sedum sediforme, Anarrhinum laxiflorum, Asperula aristata subsp. aristata and Taraxacum palustre) and Carex vesicaria from the catalog of the Parque Nacional de las Illas Atlánticas.

Key words: vascular flore, chorology, ecology, Galicia, Spain.

\section{INTRODUCCION}

Desde 1984 venimos publicando nuestras aportaciones a la flora de Galicia (Rigueiro Rodríguez \& Silva-Pando 1984, Silva Pando et al. 1987, Gómez Vigide et al. 1989, García Martínez et al. 1991, Valdés Bermejo et al. 1995, Silva Pando et al. 2000, Gómez Vigide et al. 2006, Gómez Vigide et al. 2007, Silva-Pando et al. 2010) hasta llegar a los 559 taxones comentados. Hemos añadido al catálogo de la flora de Galicia, 60 nuevos taxones y hemos eliminado del mismo, 5, todo ello gracias a la colaboración de 18 investigadores. Con esta nueva entrega, pretendemos seguir aportando información florística relevante para la región gallega. En cuanto a la nomenclatura taxonómica seguimos a Flora iberica para las familias publicadas y a Flora Europaea u otras fuentes actualizadas para el resto. En este trabajo, seguimos el orden establecido por el proyecto Flora iberica en la exposición de las familias. Aquellas que no aparezcan en dicho proyecto, se sitúan al final.

\section{MATERIALES Y MÉTODOS}

Como en otras ocasiones, la metodología empleada consistió en salidas al campo, herborización, secado y etiquetado del material y revisión de pliegos en diferentes instituciones. Los materiales recolectados por los autores han sido incluidos en el Herbario LOU (Lourizán, Pontevedra), USP (Madrid) y herbarios particulares de X. R. García Martínez (GM) y F. Gómez Vigide (GV), enviándose, en su caso, copias a otros herbarios.

Para cada cita, los datos son los escritos en la etiqueta del pliego o los de nuestras libretas de campo. En aquellos en los que no consta algún dato de municipio o georreferencia en la etiqueta del pliego, la hemos añadido nosotros, indicándolas entre corchetes. En los mapas hemos utilizado como icono para las citas basadas en referencias bibliográficas o de revisión de pliegos, un círculo, mientras que para las citas nuevas aportadas en este trabajo el icono es una estrella. La determinación se ha realizado mediante las floras básicas, monografías u otros trabajos que figuran en la bibliografía. Las especies del género Taraxacum han sido determinadas siguiendo a Galán (2010).

\section{RESULTADOS Y DISCUSION} Ranunculus peltatus Schranck subsp. peltatus var.
peltatus

PORTUGAL: Trás os MonTes: Bragança, Samil, 29TPG8627, $700 \mathrm{~m}$, arroyo sobre serpentinas, 3-VI-1989, F. J. Silva-Pando 5606 y G. B. G., LOU 14924.

De acuerdo a Cook et al. (1986: 291) es novedad para el noreste trasmontano portugués.

Modiola caroliniana (L.) G. Don

ESPAÑA: PonTevedra: Tomiño, Santa María, 29TNG2048, 30 m, en márgenes del río Pego, 11-V-2010, J. B. Blanco-Dios, LOU 34983.

La única cita que conocemos corresponde a Romero Buján \& Amigo (1993: 199) de Padrón (A Coruña) (cf. Romero Buján 2008: 93). La presencia de esta leguminosa en el sur de Galicia confirma las sospechas de Romero Buján (1.c.). Novedad para Pontevedra.

\section{Clypeola jonthlaspi L. subsp. jonthlaspi}

ESPAÑA: Pontevedra: Tui, Caldelas, en la margen del Miño, [29TNG35], B. Merino, Herbario I. Sánchez Cantón 972.

Interesantísima cita cuyas localidades más próximas están en las provincias de Segovia y Burgos (Morales 1993: 201s). Los frutos en silícula latisepta, monospermos, orbiculares $(4 \times 4 \mathrm{~mm})$ y escotados no dejan lugar a dudas. Véase su iconografía en la figura 2 de la tab. 34 de Cavanilles (1791: 106). Merino (1901b: 198) la citó de "una isleta del Miño frente á Caldelas" pero no la incluyó en su Flora de Galicia; sí menciona el género, con asterisco, en la clave de la familia, indicando así que se trata de un género no encontrado hasta el presente en Galicia (Merino 1905: 99). Lo más probable es que Merino recordase haber visto y herborizado la planta, de ahí que incluyera la mención al género en la clave, aunque al no disponer del 
material no se decidió a recogerla en el catálogo, sin duda porque habría olvidado que remitiera el pliego al instituto de Pontevedra.

\section{Iberis ciliata subsp. contracta (Pers.) Moreno}

I. contracta Pers.- I. linifolia auct.

ESPAÑA: OURENSE: Rubiá, Sierra da Enciña da Lastra, As Galegas, 29TPH7208, $1022 \mathrm{~m}$, sobre roquedos calizos, 26-VI-2009, F. J. Silva-Pando 15074, R. Pino, J. J. Pino et al., LOU 34565, 34568.

Después de lo dicho en Silva-Pando et al. (2010: 42), vemos por un lado la cita de Costa et al. (1984: 170) para la cercana localidad de Covas, en el mismo macizo que la nuestra. Sobre la otra localidad provincial, véase SilvaPando et al. (1.c.). Por otro lado, Carrasco et al. (2005: 27) la señalan de manera sorprendente de los arenales y acantilados de Ponteceso (A Coruña). Revisado el pliego, gracias a la amabilidad de Emilia Redondo, del herbario $\mathrm{MACB}$, pudimos comprobar que las semillas eran todas aladas, si bien de extensión reducida y no mucíferas, lo que confirmaba nuestras sospechas de que podría tratarse de I. procumbens Lange. Aprovechemos la ocasión para rescatar del olvido las citas de Rivas Mateo (1899: 102) de I. amara L. e I. linifolia L. de la Illa de Arousa, allá por el mes de julio de 1898 y de las que afirma haber sido recogidas en sus excursiones y no citadas en la Flora gallega del Sr. Planellas. En el herbario de la Facultad de Farmacia de Madrid (MAF) no se conservan testimonios del autor (José Pizarro, com. pers.) por lo que habrán de olvidarse de nuevo.

\section{Crassula arborescens (Mill.) Willd.}

ESPAÑA: Pontevedra: Bueu, Isla de Ons, dunas de la playa de Canexol, 29TNG0591, 15 m, 2-VIII-2008, R. Pino, J. J. Pino \& F. J. Silva-Pando, LOU 33111.

Naturalizada en las pequeñas dunas secundarias y escapada sin duda de las viviendas cercanas. Novedad para la flora de Galicia. Por parte de la dirección del Parque Nacional de la Illas Atlánticas debería estudiarse su eliminación.

Sedum dendroideum Moc. \& Sessé ex DC.

ESPAÑA: A CoRUÑa: Ribeira, playa de Corrubedo, Aguiño, A Graña, 29TMH9709, 15 m, en escombros abandonados en las dunas terciarias, bajo un pequeño rodal de Pinus pinaster, 30-I-2011, R. Pino \& J. J. Pino, LOU 35020.

Citado por Pino et al. (2010: 389) de las provincias de Ourense y Pontevedra, aparece ahora en una zona de residuos en el límite del parque natural del Complexo Dunar de Corrubedo e Lagoas de Carregal e Vixán. Aunque se trata de una planta de crecimiento lento y poco invasiva, su presencia en el perímetro del LIC, hace aconsejable su erradicación. Novedad para A Coruña.

\section{"Sedum sediforme (Jacq.) Pau"}

La especie se citó por primera vez para Galicia en un inventario de Linario glabrescentis-Cheilanthetum tinaei de la localidad de Espandariz (Quiroga, Lugo) en Fernández et al. (1987: 210). Uno de los autores (T. González, com. pers.) nos indicó que de existir pliego, en el inventario realizado en Espandariz, debía conservarse en LEB, pero consultado éste, no parece haber testimonio alguno. Niño et al. (1994: 74), recogen en su catálogo la anterior referencia junto con la de Bellot (1968). No obstante, Bellot, en esa publicación menciona 5 especies de Sedum (S. acre, S. anglicum, S. brevifolium, S. dasyphyllum, S. villosum) entre las que no vemos a la nuestra. Posteriormente, Castroviejo \& Velayos (1997: 148) indican en la secuencia provincial, «Lu», sin paréntesis, por lo que ha de entenderse que revisaron pliego de la provincia. En MA tampoco pudieron darnos referencias del pliego que hubo de servir de base para la secuencia provincial de Flora iberica y no se conserva la lista de pliegos revisados. Souto \& de Sá (2005: 79) dicen haberla encontrado en la isla de Ons (Bueu, Pontevedra). No hemos podido revisar el pliego, aunque la fotografía que ilustra la cita referida se refiere a Crassula arborescens (Mill.) Willd. Olvidemos también la sinonimia que allí se nos da. Finalmente, Romero Buján (2008: 46) mantiene como referencias en Galicia de la especie, la original de Fernández et al. (1.c.) y naturalmente a Flora iberica.

Todas las referencias de las que disponemos hasta ahora no son fiables o comprobables, por lo que se excluye de momento del catálogo gallego.

\section{Potentilla asturica Rothm.}

P. recta subsp. asturica (Rothm.) Mariz.- P. hirta var. stricta subvar. brevidentata Merino, Brotéria, Sér. Bot. X: 189 (1912) [Typus: Habita con la var. en los montes de Ramilo en el paraje llamado Choza] "var. brevidentata (v.n.) montes de Ramilo Orense". Lectotypus designado aquí: LOU 0539/2. 
ESPAÑA: OURENSE: Carballeda, Fonte da Cova, 29TPG8688, $1800 \mathrm{~m}$, en pizarras, 20-VII-1987, F. J. Silva-Pando, M. C. Casalderrey et al., LOU 09447; ibidem, Peña Trevinca, pr. Fonte da Cova, 29TPG8687, 28-VII-1991, F. Gómez Vigide, GV 7057; A Veiga, A Ponte, supra Lagoa da Serpe, 29TPG7778, $1850 \mathrm{~m}$, en pastizal derivado de pizarras, 14-VIII-2008, F. J. Silva-Pando 14821, LOU 35403; ibidem, A Ponte, 29TPG7779, $1120 \mathrm{~m}$, camino de los prados, 2-VIII-1984, F. Gómez Vigide, GV 7059; A Veiga, Valdín, Ladeira, 29TPG6875, 1265 m, 5-VIII-2009, X. R. García Martínez, F. Gómez Vigide y F. J. SilvaPando, GM 8611; Viana do Bolo, Pradorramisquedo, a orillas del río Bibei, 29TPG6868, 28-VII-1973, F. Gómez Vigide, GV 7075; ibidem, Pradorramisquedo, 29TPG6866, 1105 m, 5-VIII-2009, X. R. García Martinez, F. Gómez Vigide y F. J. Silva-Pando, GM 8599; ibidem, Cabezo Grande, 29TPG6766, 23-VII-1996, F. Gómez Vigide, GV 7060.

Endemismo ibérico noroccidental, cuyas localidades gallegas son Ramilo (Merino 1905: 458, sub. P. recta; l.c.) y Fonte da Cova y alrededores (Laínz 1968: 10s, SilvaPando 2008: 113). Nuestra localidad de la Lagoa da Serpe se encuentra entre ambas. Estos últimos años la hemos visto abundante en el municipio de A Veiga, por encima de $1000 \mathrm{~m}$. En el Herbario Merino, depositado en LOU, hay 2 pliegos, pero el designado aquí es el que mejor se ajusta a la descripción y tiene la anotación "v.n.".

\section{Alchemilla glabra Buser}

\section{A. vulgaris subsp. alpestris (F.W. Schmidt) Murb.}

ESPAÑA: Lugo: Folgoso del Caurel, As Cruces, 29PPH6018, 1270 m, calizas Mesobromion, 3-VII-1993, F. J. Silva-Pando 9142, V. Rodríguez Gracia y P. González Hernández (LOU 20425, 20426); Samos, "Portela-Peñabolosa" [29TPH42], s/f, Merino (sub. A. vulgaris) y Samos-Seoane do Courel, "Regatos de Portela-y de la Rogueira", [29TPH52], s/f., Merino, LOU 488/2 p.p. sub A. vulgaris var. subsericea; Samos, "Montes de Portela encima del valle de Loúzara-Lugo/ $L$. P. M." [29TPH42], LOU 00488/1 sub var. subsericea Gren". OurENSE: Viana do Bolo, Montes de Ramilo, [29TPG67], s/f, B. Merino S. J., LOU $00487 / 4$ p.p.

El primero en citar la especie fue Merino (1905: 423s, sub. A. vulgaris; 1912: 186, sub. A. vulgaris ssp. alpestris), aunque de acuerdo a su herbario, los pliegos LOU 488/1,2 corresponden a nuestra especie, mientras que los LOU-00487/4 y 488/2, de "[OURENSE: Viana do Bolo] Montes de Ramilo, s/f.", contienen también muestras de A. coriacea (Rev. Laínz; Laínz 1955: 119, 1971: 10), aunque en este caso no descartamos una mezcla de materiales de distintas localidades. Más reciente es la cita de Silva-Pando (1994: 313) para la Sierra de Ancares. Romero Buján (2008) no la incluye entre las alchemillas gallegas, a pesar de la citas anteriores, llevando todas las de A. vulgaris a A. xanthochlora, siguiendo a Walters (1968: 57), aunque éste incluía $A$. alpestris auct. en $A$. glabra. Fröh- ner (1998: 234s) tampoco la cita para la región, además de llevar alpestris a nuestra especie. Las muestras indicadas por Laínz (1971: 10), que por su indumento se separaban de la subser. Glabrae Rothm., las llevamos igualmente a nuestra especie.

\section{Alchemilla saxatilis Buser}

A. alpina subsp. saxatilis (Buser) É. G. Camus in Rouy \& É. G. Camus

ESPAÑA: OURENSE: Carballeda, Trevinca, 29TPG8686, $1820 \mathrm{~m}$, en suelos de distinto grosor en la proximidad de afloramientos rocosos de cumbre, 2-VII-2002, Í. Pulgar \& J. Izco, SANT 47347 sub A. transiens (Buser) Buser; ibidem, Fonte da Cova, 29TPG8687, $1700 \mathrm{~m}$, cervunal, 3-VIII-1990, F. J. Silva-Pando 7047 \& G. B. G., LOU 21398; ibidem, Fonte da Cova, 29TPG8688, 1800 m, en pizarras, 20-VII-1987, F. J. Silva-Pando, M. C. Casalderrey et al., LOU 09445; ibidem, Peña Trevinca, Peñas Longas, 29TPG8687, $1800 \mathrm{~m}$, en roquedos, 08-VII-2006, R. Pino, J. J. Pino, J. L. Camaño, F. J. Silva-Pando, F. Gómez Vigide, X. R. Garcia, J. González, Alberto, A. Pino \& R. Pino, LOU 30888; Carballeda, Fonte da Cova, 29TPG8484, 1700-1800 m, pizarras, 28-VII1983, X. R. García Martinez 33/4, LOU 24544; ibidem, Fonte da Cova, 29TPG8485, $1800 \mathrm{~m}$, gleras sobre xistos, 28-VIII-1986, X. R. García Martínez 284, LOU 24547; ibidem, Fonte da Cova, 29TPG8484, 1700 $1800 \mathrm{~m}$, pastos sobre esquistos, 3-VIII-1990, X. R. García Martínez 4698, Silva-Pando \& G. B. G., LOU 24541; ibidem, alrededores de Trevinca Norte, 29TPG8380, 1900 m, 17-VII-1984, S. Ortiz, SANT 16585; ibidem, refugio de Trevinca, 29TPG88, $1750 \mathrm{~m}, 27-\mathrm{V}-1983$, SANT 16320; ibidem, Pena Surbia, 29TPG8280, 2095 m, 5-VII-1980, F. Gómez Vigide, GV 6970; A Veiga, sobre la laguna de A Serpe, 29TPG7877, c. 1700 m, 3-VIII-1984, F. Gómez Vigide, GV 6972; ibidem, A Ponte, Lagoa da Serpe, 29TPG7877, 1700 m, sobre esquistos, 6-VIII-2009, F. J. Silva-Pando 15363, F. Gómez Vigide \& X. R. García Martinez, LOU 36849; ibidem, A Ponte, bajo el Fial, supra Lagoa da Serpe, 29TPG7778, $1780 \mathrm{~m}$, en repisas y fisuras de esquistos, 14-VIII-2008, F. J. Silva-Pando 14830, LOU 36441. ZARAG0ZA: Tarazona, Parque Natural de la Siera del Moncayo, pr. Ermita de la Virgen del Moncayo, 30TWM9727, $1600 \mathrm{~m}$, sobre roquedos esquistosos frescos, 9-VII-2009, F. J. SilvaPando 15121, R. Pino y J. L. Camaño, LOU 36851.

Merino (1912: 182) lleva a esta especie las plantas de Ancares (Merino 1905: 424, sub. A. alpina), confirmado por Silva-Pando (1994: 313; Silva-Pando et al. 2010: 42), quién aporta varios pliegos nuevos. Aedo et al. (2000: 36) reconocen la determinación de Merino, aportando un pliego. Romero Buján (2008: 109) la indica de Lugo y Ourense, aunque en la referencia de Laínz (1971: 10s), éste sólo recordaba la cita de Merino (1912: 186), no indicando localidad concreta. De Ourense fue indicada por Fröhner (1998: 326s), aunque la única localidad concreta (Peña Trevinca) la dan Pino et al. (2008: 26, LOU 30888!). Seguimos sin saber a qué corresponde la planta de Peña Trevinca citada por Laínz (1971: 10s, sub A. transiens) después de lo dicho por Aedo et al. (2000: 36) en su 
comentario sobre nuestra especie. Romero Buján (2008: 108) señala que estos últimos autores citan la especie para Ourense, cuando sólo se refieren a A. transiens (Buser) Buser.

\section{Alchemilla transiens (Buser) Buser in Dörfl.}

\section{A. basaltica Buser, nom. illeg.}

ESPAÑA: LEóN: Candín, Tejedo, Ancares, 29TPH8146, 12-VII1993, J. Guitián, M. Guitián, Í. Pulgar \& Gómez de Azcárate, SANT 63166 sub A. saxatilis Buser. Lugo: Cervantes, Pico Tres Obispos, 29TPH7441, 1770 m, 22-VIII-1985, F. J. Silva-Pando 2853, LOU 11820. OuRENSE: Carballeda, alto de Peña Trevinca junto al albergue, 29TPG8687, $1780 \mathrm{~m}$, pizarras, 28-VII-1983, F. J. Silva-Pando \& G. B. G., LOU 13997; ibidem, Macizo de Peña Trevinca, Pico Sestil, 29TPG8687, 1850 m, 25-VII-1991, F. Gómez Vigide, GV 6973; ibidem, entre Refugio de Trevinca y A Escrita, 29TPG8584, 1800-1880 m, 1VII-1983, S. Ortiz, SANT 21541. Zamora: Porto, Valle la Frigina bajo Pico Moncalvo, 29TPG7974, 1750 m, 19-VII-1992, F. J. Silva-Pando 8069, LOU 20390. Zaragoza: Tarazona, Parque Natural de la Sierra del Moncayo, pr. Ermita de la Virgen del Moncayo, 30TWM9727, 1600 $\mathrm{m}$, sobre roquedos esquistoso frescos, 9-VII-2009, F. J. Silva-Pando 15120, R. Pino \& J. L. Camaño, LOU 36850, 36582; ibidem, Parque Natural de la Sierra del Moncayo, supra Santuario Virgen del Moncayo, 30TWM9626, 1900-2000 m, sobre pizarras y esquistos, 27-VI2008, F. J. Silva-Pando y R. Pino, LOU 35425; ibidem, 29TWM9626, 2000-2200 m, sobre pizarras y esquistos, 27-VI-2008, F. J. Silva-Pando y R. Pino, LOU 35390.

Laínz (1971: 10) la indica de la "Zona de Peña Trevinca, dentro de Orense. Y predomina, por lo menos, en la zona de Lugo [Picos de Ancares]...", aunque después parece retractarse de la misma (cf. Aedo et al. 2000: 36), en base a Fröhner (1998: 333s). Silva Pando (1994: 312, sub. A. basaltica) la cita de la Sierra de Ancares, aportando además un pliego del herbario del Jardín Botánico de Madrid (MA 178437) revisado por H. Kalhebas en 1973. Sus características son la presencia de 5-(6-7) lóbulos, con el lóbulo central de las hojas basales dividido menos del $100 \%$ y los dientes de los lóbulos delgados y estrechos, claramente más largos que anchos. Al no estar incluida en el Catálogo de la Sierra de Moncayo (Uribe \& Zorrakin 2004), donde si lo estaba la especie anterior pero de forma genérica, aprovechamos para incorporar la cita arriba señalada, donde convive con $A$. saxatilis, tal como ocurre en Peña Trevinca.

\section{Teline monspessulana (L.) K. Koch}

ESPAÑA: PonTEvEdRA: Pontevedra, Lourizán, 29TNG2795, 20 $\mathrm{m}$, en matorral, 23-II-2007 J. B. Blanco-Dios, LOU 34992, 34993.
Novedad para Galicia. Talavera \& Gibbs (1999: 143) la señalan como presente en el S y E de la Península Ibérica. También la hemos visto en Salceda de Caselas (Pontevedra) lo que resulta coherente con las poblaciones señaladas para Minho (Portugal).

Pisum sativum subsp. elatius (M. Bieb.) Asch. \& Graebn.

ESPAÑA: OURENSE: Rubiá, Monte Páramo, camino dela Barca, 29TPH7904, en sustrato calizo, 9-VI-1983, F. Gómez Vigide, GV 5156, SEV 159964 (rev. C. Romero Zarco, II-2000); Cartelle, A Lomba, río Arnoia, 29TNG7375, $200 \mathrm{~m}$, en zona ruderal, 21-V-2009, R. Pino \& A. Pino, LOU 34420.

Romero Zarco (1999: 485) no incluye provincia alguna de Galicia en la secuencia corológica, por más que ya lo advirtieran Aedo et al. (2000: 49). Efectivamente, Merino (1905: 339) sub P. elatius Bieberstain decía haber visto "algunos pies entre los sembrados en las haciendas del Incio, Lugo". Del oriente ourensano la señalaron Giménez \& Amigo (1996: 112). Ahora aparece en laderas de solana del cañón del río Arnoia, en ambiente termófilo de Rusco aculeati-Quercetum roboris var. Quercus suber. Nuestra planta así como los dos pliegos de Merino (LOU $374 / 1$ y 374/2) se adscriben a la var. elatius, con pedúnculos más largos que las hojas. Naturalmente, lo dicho por Romero Buján (2007: 121, 2008: 85) habrá de cambiarse.

\section{Hippocrepis comosa L. s.l.}

ESPAÑA: A CoRuÑa: Cariño, Sierra de la Capelada, Piedras del Cadro, 29TNJ84, $50 \mathrm{~m}$, en pastizales eutrofos sobre ecoglitas, 2-VII2007, R. Pino, J. J. Pino \& F. J. Silva-Pando LOU 32589; ibidem, Sierra de la Capelada, Piedras del Cadro, 29TNJ84, 50 m, en pastizales eutrofos sobre ecoglitas, 24-VI-2007, R. Pino, J. J. Pino, F. J. SilvaPando 11241 y A. Martínez, LOU 37214; ibidem, Cantiles de Cuadro, 29TNJ84, 100 m, sobre ecoglitas, 30-V-2007, F. J. Silva-Pando 11162, LOU 37215 .

El primero que citó el género en Galicia fue Merino (1905: 311), quién señala $H$. comosa de la provincia de Lugo. Bellot (1947: 292) considera su único pliego gallego visto (Santalla, Lugo, MA-68444) como H. comosa L. var. microphylla Rouy, caracterizada por tener foliolos muy pequeños. Laínz (1967: 21s, sub H. comosa) la señala de Ourense, además de indicar varias localidades de los pliegos de Merino (LOU), y recordaba la determinación de Pau (1916: 164) donde llevaba la planta gallega a $H$. scabra DC. subsp. commutata (Pau) Pau; al año siguiente, ese mismo autor (Laínz, 1968: 7) determina las plan- 
tas gallegas como H. commutata, incluyendo lo que Bellot (1947: 292) llamó H. comosa fma. microphylla Rouy, aunque Amigo (1984: 226) e Izco et al. (1985b: 78) aún mantienen la antigua determinación. Con posterioridad se ha vuelto a citar de Galicia por Valdés Bermejo et al. (1995: 142) y Giménez de Azcárate y Amigo (1996: 80) ya bajo la denominación correcta. La cita de Lange (1865: 375) de la localidad del Castro (León) muy próxima a Galicia, recogida en Carbó et al. (1977: 82), no resulta verosímil. Aparece ahora al fin la especie sensu latu, en los suelos ultrabásicos de la Serra de A Capelada. La planta muestra características morfológicas que la separan de $H$. comosa típica, estando a punto de publicarse un trabajo sobre la misma que aclararía su estatus taxonómico. La nuestra es, por tanto, novedad regional.

\section{Paraserianthes lophantha (Willd.) I. C. Nielsen}

Albizia distachya (Vent.) Macb.

ESPAÑA: Lugo: Foz, Playa Polas, 29TPJ3531, 12 m, en borde de cultivos, 27-VIII-2009, R. Pino \& F. Pardavila LOU 34721.

Leguminosa exclusivamente litoral en Galicia. Es utilizada como ornamental en muchas ocasiones lo que unido a su carácter invasor (Bañares et al. 2004: 1023) supone un riesgo importante para comunidades de escasa cobertura. Conocida para A Coruña desde Castro et al. (1989:109), cita que olvida Romero Buján (2007: 121). Silva-Pando et al. (2000: 23), destacan su presencia en Cangas (Pontevedra) donde efectivamente constatamos su rápida expansión, al tiempo que Pino et al. (2009: 120) la indican en el meridional municipio de Oia. Ya la hemos visto en el Parque Nacional de las Islas Atlánticas (como ornamental en la isla de Ons) donde habrá que vigilar su estatus. Novedad para Lugo.

\section{Oenothera rosea Aiton}

ESPAÑA: Lugo: Ribadeo, Playa de Os Bloques, 29TPJ5821, 5 $\mathrm{m}$, en zonas nitrificadas junto al mar, abundante, 27-VIII-2009, R. Pino \& F. Pardavila, LOU 34716.

Gómez Vigide (1985: 370) fue el primero, y que sepamos único, en señalarla para la provincia de Lugo. Su presencia en Ribadeo, siguiendo el litoral reclama la atención sobre su capacidad expansiva. Para Galicia, ya Texidor (1869: 611) denunciaba su presencia " ¿connaturalizada?" en "los muros y paredes de Santiago", en todo caso muchos años antes de lo que indica Romero Buján (2007: 122) a pesar de que Willkomm \& Lange (1880: 181) dejan bien claro la referencia a Texidor.

\section{Chamaesyce polygonifolia (L.) Small}

ESPAÑA: Lugo: Foz, Playa de la Pampillosa, 29TPJ3728, $10 \mathrm{~m}$, en dunas secundarias, muy abundante, 27-VIII-2009, R. Pino \& F. Pardavila, LOU 34724.

Citado sólo con carácter general para la provincia por Benedí (1997: 291). La nuestra es la primera cita concreta de la especie en Galicia. Destacamos su carácter expansivo en las dunas secundarias.

\section{Geranium purpureum Vill.}

ESPAÑA: A CoRuÑa: Noia, 29TNH0936, al lado de setos y muros, 8-V-1987, F. Gómez Vigide, GV 4209. Lugo: Lugo, 29TPH1864, 29-V-1991, F. Gómez Vigide, GV 4210. OuRENSE: Rubiá, sobre Cobas, 29TPH7805, en pastos de orilla, 13-VI-1984, F. Gómez Vigide, GV 4211. Pontevedra: Ponteareas, Canedo, 29TNG4270, 10-V1963, F. Gómez Vigide, GV 4212; Baiona, Sabaris, 29TNG1562, 30 m, márgenes de camiños, 31-V-2004, X. R. García Martínez 7633, GM 7633.

Rodríguez Gracia (1983: 18) la citó de Ourense como novedad regional, añadiendo "Debe ser frecuente en toda Galicia mezclada con el G. robertianum"; esta semejanza ha llevado a que la gente del pueblo da a las dos indistintamente el nombre de Hierba de san Roberto. Niño et al. (1994: 97) y Romero (2008: 62) la incluyen con referencias espúrias. En el borrador de Flora iberica [15-I-2011], tampoco aparece señalada de la provincia de Pontevedra, a pesar de la distribución del pliego. Novedad provincial para A Coruña, Lugo y Pontevedra.

\section{Calystegia silvatica subsp. disjuncta Brummitt}

ESPAÑA: A CoRuña: Carballo, arenales de Baldaio, 29TNH2894, 8-VII-2002, F. Gómez Vigide, GV 2981; Santiago, pr. Santiago, Monxoi, 29TNH4048, $300 \mathrm{~m}$, en talud sobre la carretera, 22-VI2005, R. Pino \& J. J. Pino, LOU 30597. Lugo: Lugo, As Gándaras, 29TPH1766, 15-VI-2004, F. Gómez Vigide, GV 2983. Pontevedra: Marín, Pastoriza, arroyo de Maceira, 29TNG2286, $380 \mathrm{~m}$, en margen de camino, húmedo, 1-X-2008, R. Pino \& J. J. Pino (Lou 34251); Cangas, Darbo, 0 Gatañal, 29TNG1678, 40 m, en el margen del riachue1o, 12-VIII-2005, R. Pino \& J. J. Pino, LOU 30670/1, 30671/3); Vigo, prope IES do Castro, 29TNG2376, cubrindo muros en xardíns, 12-IX2005, X. R. García Martinez 7896, GM 7896; ibidem, entre Beiramar y Marqués de Valterra, 29TNG2175, en setos y ribazos, 5-V-1999, F. 
Gómez Vigide, GV 2982; Baiona, Sabaris, paseo pola Foz do Miñor, 29TNG1562, 5 m, 24-VII-2008, X. R. García Martinez 8383, GM 8383, rev. S. Silvestre.

El material estudiado se caracteriza por las bracteolas saciformes, ligeramente más largas que anchas y el ápice obtuso con un pequeño acumen, mientras que la corola suele sobrepasar ligeramente los $50 \mathrm{~mm}$. El icono de la especie que publica Valdés et al. (1987: 370) es bastante ilustrativo. Por el contrario, los especimenes de los pliegos: PONTEVEDRA: Lalín, pr. Camposancos (Goleta), 10VIII-2007, F. Gómez Vigide, GV 2980bis y Cangas, Darbo, Sierra Poniente, 29TNG1779, $90 \mathrm{~m}$, en muros y taludes, 24-V-2003, R. Pino \& A. Pino, LOU 26115, por sus bracteolas agudas con un pequeño acumen y truncadas a ligeramente cordadas en la base, corola mayor de $50 \mathrm{~mm}$ y pedúnculos generalmente menores que las hojas, estimamos puede tratarse de un híbrido entre $C$. sylvatica y C. sepium (cf. Rodríguez \& Rodriguez, 1999: 177). Es bien sabido que fue Laínz (1974: 15, sub C. sylvatica) el primero en citarla para Galicia, de entre Lourizán y Placeres (Marín, Pontevedra), pero no Merino (1906), como erróneamente señala Romero Buján (2008: 44). Rodríguez \& Rodríguez (1999: 177, sub C. sylvatica) además de ampliar su areal a diversos municipios orensanos, cuestionan el valor del binomen por la variabilidad de los valores biométricos y sugieren que podria subordinarse a $C$. sepium (L.) R. Br., comentario que olvidan Carlón et al. (2010: 38). Posteriormente fue citada de A Coruña por Gómez Vigide et al. (2006: 58, sub C. sylvatica). Brummitt (1997: 339) limita la distribución de la subsp. disjuncta al S y E de España y Baleares en lo que respecta a la Península Ibérica. Se confirma su presencia en Galicia, siendo novedad para Lugo.

\section{Echium vulgare subsp. pustulatum (Sm.) E. Schmid \& Gams in Hegi}

ESPAÑA: Lug0: Quiroga, Río Sil, 29TPG4795, $300 \mathrm{~m}$, en canchales de río, 26-VI-2009, F. J. Silva-Pando 15046, R. Pino, J. J. Pino et al., LOU 36865. OuRENSE: Rubiá, Sierra da Encina da Lastra, Biobra, 29TPH7307, 1000 m, sobre suelos calizos, 27-VI-2009, F. J. Silva-Pando 15068, R. Pino, J. J. Pino et al., LOU 36866; ibidem, Sierra de Encina de la Lastra, Picos de Oulego, Pena Tallada, 29TPG7004, 900 m, 12-VI-2009, F. J. Silva-Pando 14947, X. R. García Martínez y A. Guitián, LOU 36862.

La subespecie pustulatum soló está citada para La Rúa-Orense por Merino (1906: 156s), mientras que Valdés Castrillón [Borrador Flora Iberica, 28-VII-2010] la indica de Lugo y (Ourense). Gimenez \& Amigo (1996: 61) sólo mencionan la subespecie típica. Para Lugo sería la primera localidad publicada.

\section{Myosotis congesta R. J. Shuttlew}

ESPAÑA: Pontevedra: Marín, Seixo, a ponte de Guimeráns en la orilla del río Loira, 29TNG2290, 10 m, pie de muro, 30-IV-2008, M.J. Rozados \& S. González Pazos 238, LOU 36388.

En el borrador del género para Flora iberica [Valdés Castrillón, 14-VII-2009] aparece indicada para la provincia de A Coruña, pero no hay ninguna cita publicada para la región.

\section{Verbena bonariensis L.}

ESPAÑA: PoNTevedRa: Pontevedra, Lourizán, frente a la puerta principal del CIF, 29TNG2795, $10 \mathrm{~m}$, en suelos nitrificados de borde de carretera, VI-2009, J. B. Blanco-Dios, LOU 34982.

Pujadas \& Plaza (2010: 15s) la indican del Pais Vasco y Cataluña, mientras que en Portugal asciende hasta Douro Litoral. La localidad española más cercana es El Astillero (Cantabria) (Aedo et al. 1985: 205). Como las demás citas ibéricas, es planta alóctona. Novedad regional.

\section{Glechoma hederacea L.}

ESPAÑA: A CoRuÑa: Cedeira, A Veiga, entre Viduedo y A Cruz, puente sobre el ríoForte, 29TNJ8638, $250 \mathrm{~m}$, serpentinas, X. R. García Martñinez \& U. García Aldao, GM 4337; Ortigueira, Mera, 29TNJ8733, 40 m, 10-V-1994, X. R. García Martínez, A. Prunell \& X. Soñora, GM 6074; Betanzos, Piadela, Guiliade, nas marxes do río Mero, 29TNH6189, 40-45 m, 1-IV-2009, M.J. Rozados \& S. González Pazos, LOU 37101; Carral, Herves, Beira, na ribeira do río Barcés, 29TNH5284, 80-85 m, 1-IV-2009, M.J. Rozados \& S. González Pazos, LOU 37102; Boqueixón, rego sobre regato Freixido, 29TNH4443, 200 $\mathrm{m}$, ripisilva con Fraxinus angustifolia, 15-V-1989, X. R. Garcia Martínez y A. Prunell, GM 3768; Noia, A Barquiña, 29TNH0938, en pastos de ribera, 12-IV-1988, F. Gómez Vigide, GV 4410. Pontevedra: Marín, Allariz, molino, 29TNG2388, $180 \mathrm{~m}$, en prados de siega, 24-II-2008, S. Rial \& D. Álvarez, LOU 32795; Redondela, cerca de Cedeira, 29TNG3118, 22-VII-1964, F. Gómez Vigide, GV 4413; Ponteareas, Canedo, 29TNG4270, 15-VI-1974, F. Gómez Vigide, GV 4474.

Merino (1906: 202) la señaló como abundante "en parajes sombríos de todas las provincias galaicas" sin dar localidades precisas. Parece que Niño et al. (1994: 101) y Romero Buján (2008: 78) se basaron en dicho aserto para indicarla de todas las provincias gallegas, aunque los primeros decidieron acertadamente añadir un interrogan- 
te a las provincias de Ourense y Pontevedra. Buch (1951: 66) apunta también a todas las provincias sin que veamos material de respaldo. Efectivamente no encontramos referencias bibliográficas de Glechoma en Pontevedra ni pliegos en el herbario de Merino que avalen su presencia en esa provincia. Consideramos pues nuestra cita como primera para la provincia.

\section{Linaria aguillonensis (García Mart.) García Mart. \& Silva Pando \\ Linaria polygalifolia subsp. aguillonensis (García Mart.) Castrov. \& Lago}

ESPAÑA: Lugo: Galdo, B. Merino s/d, LOU 812/1 sub L. lusitanica; Arenales de San Ciprián, B. Merino, s/d, LOU 812/2 sub L. lusitanica; Xove, Xuances, Caranza, praia do Esteiro, 29TPJ1641, 10 m, 23-VII-1994, M. Castro, L. Freire, X. R. Garcia Martinez 6197, Gonzalez, F. J. Silva-Pando, F. Gómez Vigide \& GBG, GM 6197; Barreiros, Tupido, San Bartolomé, 29TPJ4225, 5 m, 24-VII-1994, M. Castro, L. Freire, X. R. Garcia Martínez 6239, Gonzalez, F. J. Silva-Pando, F. Gómez Vigide \& GBG, GM 6239.

Una relectura de Merino (1902: 85) nos induce a revisar los pliegos de lo que llamó en su Flora Linaria caesia var. broteri (Merino 1906: 76) y sinonimizó (in sched.) a Linaria lusitanica (Brot.) Hoffmans. \& Link, e incluso a Linaria lamarckii Rouy. Vemos que ANTHOS [03-022011] cae en similar error. Laínz (1971:21) llamó la atención sobre estas formas latifolias del Norte gallego, pero no supo darle la interpretación correcta. Novedad provincial para Lugo, considerada hasta el momento endemismo coruñés (Castroviejo \& Lago 1990, Sáez \& Bernal 2009).

\section{Linaria lamarckii Rouy}

Linaria polygalifolia subsp. lamarckii (Rouy) D. A. Sutton

PORTUGAL: BeIRA LITORAL: Aveiro, praia da Barra, $10 \mathrm{~m}$, trasduna, 02-V-2009, A. Aldao, F. Ferrón, I. García Bernadal, X. R. Garcia Martínez 8418, A. Guitián \& M. Rodríguez, GM 8418.

Las floras más al uso (Sáez \& Bernal 2009: 254, Valdés et al. 2000) consideran que su distribución alcanza la Estremadura portuguesa, desde "la Bahía de Setubal hasta la desembocadura del Guadiana, llegando a Huelva", donde se llegó a extinguir. Nuestra cita extiende $50 \mathrm{Km}$ hacia el norte el área de distribución conocida deste endemismo luso-hispano. En cuanto a la única población española, recientemente recuperada, ver Plaza Arregui \& Rodríguez Hiraldo (2009).

\section{"Anarrhinum laxiflorum Boiss."}

Citada por Benito (1995: 10) de Avesedo de Donís (Cervantes, Lugo) a $1050 \mathrm{~m}$, el 27-VI-1994. Puestos en contacto con el conservador del herbario JACA, nos remitió una imagen del pliego JACA 111094, comprobando que se trataba de A. bellidifolium (L.) Willd. Especie, pues, a eliminar del catálogo regional.

Asperula aristata subsp. scabra (J. Presl. \& C. Presl. ex Lange) Nyman

A. aristata var. laevis Lange

ESPAÑA: Lugo: Folgoso do Caurel, monte Cereixedo, al sur de Visuña, 29TPH5818, 14-VII-1997, F. Gómez Vigide, GV 7322; ibidem, entre Ferramulín e Visuña, 29TPH5917, 900 m, calías, 27-VII-1992, X. R. Garcia Martínez 5299, I. Díaz \& F. J. Silva-Pando, GM 5299; ibidem, Visuña, 29TPH5819, pasto calizo, 2-VIII-1989, J. Giménez \& J. Amigo, SANT 27307, sub A. cynanchica L.; ibidem, Caurel, As Cruces, 29TPH6018, 1300 m, 31-VII-1989, J. Amigo \& J. Giménez, SANT $26598 \mathrm{sub}$ A. aristata L. fil.; ibidem, Valle de Visuña, 29TPH5819, 21VII-1981, SANT 16048, sub A. cynanchica L.; Quiroga, entre Soldón y Montefurado, Río Sil, 29TPG4795, 300 m, en canchales de río, 26VI-2009, F. J. Silva-Pando 15041, R. Pino, J. J. Pino et al., LOU 36847. Pontevedra: [Tui], Caldelas, [29TNG35], Rivera del Miño entre los cascajos, 1999 (?), B. Merino LOU 01062/1 sub A. cynanchicum L. var. typica Rouy; ibidem, B. Merino LOU 01062/2 sub A. cynanchica; ibidem, B. Merino LOU 01062/3 sub A. cynanchicum typica; ibidem, $B$. Merino LOU 01062/4 sub A. cynanchicum; Tui, Salceda de Caselas, 29TNG3656, $15 \mathrm{~m}$, areas e pedregais a beiras do Miño, 9-VII-1996, X. R. Garcia Martinez 6562 \& J. González Dominguez, GM 6562: Tui, Caldelas de Tui, 29TNG3656, $15 \mathrm{~m}$, areas e pedregais á beiras do Miño, 5-VIII-1997, X. R. García Martínez 6870 \& A. Aldao, GM 6870; As Neves, baixo Chan de Vide, 29TNG5159, $20 \mathrm{~m}$, areas e pedreiras á beiras do Miño, 11-IV-1998, X. R. García Martínez 6907 \& F. J. Silva Pando, GM 6907; Arbo, Estación de Sela, 29TNG5360, 55 m, en solos pedregosos nas ribeiras do Miño, 23-V-1998, J. Amigo, R. I. Louzán, L. G. Quintanilla et D. G. San León, SANT 39712/1 sub A. arista$t a$ L. fil.; entre Sela y Las Nieves, 29TNG55, 2-VI-1950, Bellot, SANT 4976, "nueva para Galicia", sub A. cynanchica subsp. aristata. Z $\mathbf{Z}_{\mathbf{A}}$ MORA: Corrales, 30TTL7182, 9-VII-1946, Casaseca, SANT $4203 \mathrm{sub}$ A. aristata L. var. macrosiphon Wk. Prod. II p. 302; Corrales del Vino, 30TTL7182, 16-VII-1951, Casaseca, SANT 5590 sub A. cynanchica L. subsp. aristata L.; Corrales, 30TTL7182, 15-VII-1947, Casaseca, SANT $6048 \mathrm{sub}$ A. cynanchica L. subsp. aristata (L. fil.) Béguinot. PORTUGAL: Trás-Os-Montes: Bragança, Samil, $700 \mathrm{~m}$, serpentinas, 25-VI-1988, X. R. García Martínez 3388, R. Medina, F. J. Silva-Pando \& E. Valdés-Bermejo, GM 3388.

Guitián et al. (1985: 198) la citan como novedad para Ourense, a la vez que recuerdan la cita de Willkomm (1893: 131, sub var. laevis Lange) para Pontevedra. Benito (1995: 11) la herboriza en Pena Tallada (Rubiá, Ourense). Romero Buján (2008: 113) cita para Galicia nuestra planta y la 
subespecie aristata, cuando ya Ortega Olivencia \& Devesa (2007: 39s) sólo recogen en la zona la subespecie scabra. La "Asperula cynanchica L." de las calizas orensanas (Giménez de Azcárate \& Amigo, 1996: 31s) y A. aristata L. fil. de la misma zona (Fernández Areces, 1989) las llevamos a nuestra subespecie. En Galicia ha de olvidarse la subespecie nominal. Una vez vistos los materiales de LOU y SANT, comprobamos que el tamaño y forma de la corola presentan una cierta variabilidad (Ortega Olivencia \& Devesa, 2007: 41), desde 3 a $6 \mathrm{~mm}$ de largo y el tubo de netamente cilíndrico a más o menos cónico, aproximándose algo al de cynanchica. Novedad provincial para Lugo.

\section{Asperula cynanchica L. subsp. cynanchica}

ESPAÑA: LeóN: Puerto del Pontón, 30TUN352774, $900 \mathrm{~m}$, pared caliza, 30-VII-1997, F. Llamas, M. E. García \& E. de Paz, SANT 38956/1. OURENSE: Piedras entre Montefurado y la Rúa, 29TPG59, VI1942, Losa, SANT 4205 (Figura 5). OviEdo: Vega de Rengos, 880 m, en comunidad de herbazal sobre caliza en contacto con un hayedo, 27VI-2006, J. Amigo \& M.R. Guitián, SANT 56045.

Merino (1906: 271) cita la especie de Caldelas de Tuy (Po), Cortegada, Ribadavia y Barbantes (Or), pero el material se adscribe a la especie anterior. En LOU no hemos encontrado pliego que respalde la var. maritima Gren. \& Godron de Ferrol (A Coruña) en Merino (1.c.). Nuestra cita representa novedad para Ourense, en una localidad muy cercana a la nuestra de A. aristata subsp. scabra de Quiroga $(\mathrm{Lu})$, quedando pendiente de confirmar su presencia en Ferrol.

\section{Valeriana tripteris L. subsp. tripteris}

ESPAÑA: Lug0: Pedrafita do Cebreiro, monte Faro, 29TPH6022, c. 1550 m, F. \& F. Gómez Vigide, 23-VI-1993, GV 8864 (Figura 1).

Para disipar las "viejas dudas" de Laínz (1955: 141), reiteradas recientemente en Carlón et al. (2010: 60), sobre si la especie V. tripteris alcanza efectivamente las montañas gallegas y cantábricas, publicamos de buena gana copia del pliego correspondiente al ejemplar reseñado, herborizado por los hermanos Fermín y Feliciano Gómez Vigide en los aledaños del Monte Faro, en donde convive con $V$. montana, que es más abundante. Al ver en Flora iberica la sigla Lu (Lugo) pensamos que los autores tendrían buenas razones para indicarla, por lo que nuestra herborización no ofrecería novedad relevante al ser publicada. Pero al conocer ahora las reiteradas dudas de tan in-

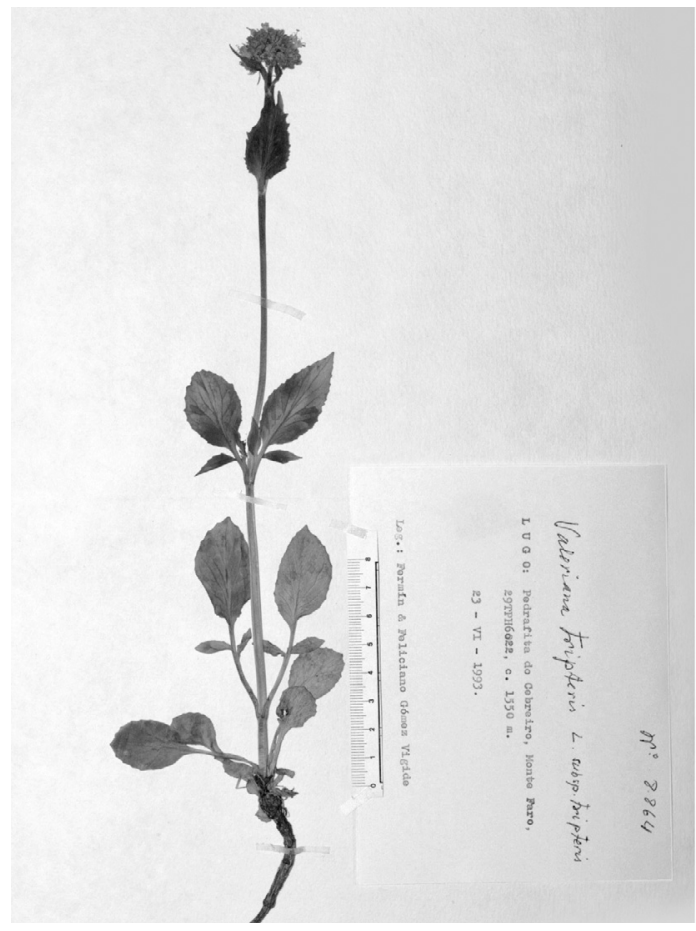

Fig. 1- Valeriana tripteris L. subsp. tripteris GV 8864.

signes botánicos la publicamos gustosos, advirtiendo además que, el que una especie no esté presente entre los 22 pliegos - de especies afines - en el herbario JBAG, no es argumento válido para negarla en otros herbarios, particulares o públicos, del territorio nacional.

Senecio pyrenaicus L. in Loefl.

\section{S. tournefortii Lapeyr.}

ESPAÑA: OURENSE: Carballeda, Peña Trevinca, entre Pico Riotorto y Mermiñeira, 29TPG8276, $1870 \mathrm{~m}$, en borde de laguna, 21-VII1987, F. J. Silva-Pando 4354, E. Valdés Bermejo et al., LOU 09579; ibidem, entre Pico Riotorto e Mermiñeira, 29TPG8276, 1950 m, pizarras. X. R. García Martínez 2627, M. C. Casalderrey, S. Martínez, P. P. Caramés, V. R. Gracia, E. Valdés, F. J. Silva-Pando \& A. Prunell, 22-VII1987, GM 2627. ZAMORA: La Baña, baixo Mermiñeira, 29TPG87, 1800 $\mathrm{m}$, beiras de lagoa glaciar, X. R. García Martínez 4714, Silva-Pando \& G. B. G., 04-VIII-1990, GM 4714.

Planta que en el momento de la recolección nos señaló E. Valdés Bermejo $(\dagger)$ pero que por olvido no incorporamos a alguna de las aportaciones anteriores de esta serie. Al Padre Merino le pasó lo mismo, como ya señaló Laínz (1956: 537), quién sacaba a la luz el olvido inexplicable de la Psoralea bituminosa L. y recordaba en su epis- 
tolario "otros descubrimientos de los que nunca dudó el Padre y a los que nunca llegó su hora en un lustro de Adiciones". No es desde luego el único caso, recordemos también la cita de Rhamnus cathartica L. en Laínz (1967: 20). La localidad más cercana la encontramos en Cabeza de la Yegua (Ponferrada, León), (Hergueta et al. 1999: 163), siendo frecuente en la Cordillera Cantábrica (Asturias y León, entre otras). Según ANTHOS [26-I-2011] fue citada por Pau (1912a) de Odollo-La Cabrera (LE), aunque en el citado artículo no aparece la especie, sino que corresponde a su trabajo Plantas nuevas de la provincia de Madrid (Pau 1912b: 39s). En este último, el autor cita S. tournefortii Lap. de la "Cordillera Cabrerica-Galicia-1872" y a continuación"La Aguiana-Odollo" recolectados por E.F.M.A. [E.F. Martin del Amo]; la Cordillera de la Cabrera, La Aguiana y Olloro se encuentran actualmente fuera de los límites de Galicia (cf. Laínz 1956: 535, 546, 548). Nieto Feliner (1985) no la señala para esta última zona.

A través de GBIF [Portal de datos GBIF, www.gbif.es. 10/I/2011. SEV, 1 registro, 10-I-2011], hemos localizado un pliego en el herbario Boutelou (SEV historico 2242-1) con la localización "Galicia", aunque por las características ecológicas de la especie y por los lugares por donde pudo herborizar Pourret, es dudoso que la hubiera herborizado en Galicia. Después de vista una fotografía del pliego, por su tallo glabro, sólo peloso en la base del capítulo, y limbo casi entero, es difícil encuadrarlo dentro de las variedades descritas por Willkomm (1870: 114s), aunque la forma angustifolia (margine obsolete denticulato) de Pau (1912b: 40) parece adaptarse bien a la planta de Pourret; ya Chater \& Walters (1976: 197) señalan que es una planta muy variable, con hojas glabras a esparcidamente crispado-pelosas por el envés y enteras o dentadas. Novedad para Galicia.

Nuestra planta no tiene que ver con el Senecio lapeyrousii Rothm., citada recintemente por Argüelles et al. (2004-2005: 168s) de la Cordillera Cantábrica.

\section{Taraxacum acutangulum Markl.}

ESPAÑA: A CoRuÑa: Muros, Louro, en la huerta de San Francisco, 29TMH9434, 20 m, en orilla de caminos, 27-III-1999, F. Gómez Vigide, GV2910.

Afín a T. ekmanii Dahlst., T. cordatum Palmgr. y T. pulchrifolium Markl. (crece en la provincia de Pontevedra), T. acutangulum se diferencia por el rostro de los aquenios más largo, sus filarias externas reflejas y el lóbulo terminal de las hojas frecuentemente alargado, lingulado. En la Península Ibérica, el resto de las localidades se encuentra sobre todo en el este y noreste. Novedad regional.

\section{Taraxacum braun-blanquetii Soest}

ESPAÑA: Lugo: Cervantes, Castelo de Doiras, 29TPH6439, 680 $\mathrm{m}$, en los alrededores del castillo en xesteiras de Cytisenion multiflori sobre pizarras, 1-V-1983, F. J. Silva-Pando 362, LOU 11020.

Afín a T. rubicundum (Dahlst.) Dahlst., se diferencia de este por los aquenios que presentan el cuerpo de 3,1-4,1 $\mathrm{mm}$ con escuámulas de erosas a tricuspidadas -las erosas pueden llegar hasta la base- y espículas robustas y alargadas hacia el ápice, pirámide $0,7-1 \mathrm{~mm}$, rara vez espiculosa, rostro 5,8-8 $\mathrm{mm}$ y vilano $3,9-6,6 \mathrm{~mm}$. Novedad regional.

\section{Taraxacum cantabricum A. Galán \& Vicente Orellana}

ESPAÑA: OURENSE: Carballeda, frente al refugio de Peña Trevinca, 29TPG8687, $1770 \mathrm{~m}$, pizarras, 17-VI-1982, F. J. Silva-Pando 249, LOU 06210.

Taxon recientemente descrito de Asturias, que también fue recolectado en Peña Trevinca, pero en la parte zamorana. Novedad regional.

\section{Taraxacum drucei Dahlst.}

\section{T. lainzii Soest}

s/l.: «in reg. Littoralis commune", B. Merino, LOU 1319/4. ESPAÑA: PoNTEVEDRA: Lalín, pr. Galegos, 29TNH7035, $500 \mathrm{~m}$, en campiñas y arcenes, 10-IV-1964, F. Gómez Vigide, GV 2911; Vigo, Samil, 29TNG17, 5 m, arenales, 24-III-1973, F. Gómez Vigide, GV 2912.

Afín a esta especie son T. merinoi Soest in Brotéria, Ci. Nat. 23: 141 (1954), de los herbazales nitrófilos de Lugo y Orense y T. canariense Soest in Brotéria, Ci. Nat. 23: 139 (1954), presente entre Arbo (Pontevedra) y Humoso (Orense). Estas 3 especies forman parte de un grupo de microespecies de Gran Bretaña, Irlanda, N y NW de la Península Ibérica con el capítulo y los aquenios de menores dimensiones que en el resto de la sección Celtica. Novedad provincial.

\section{Taraxacum ekmanii Dahlst.}

s/l.: "in regione littorea", B. Merino, LOU 1319/1bis (sub. T. ekmanii Dt., Soest, 1954). ESPAÑA: Lugo: Lugo, 29TPJ16, 430 m, orillando caminos y arcenes, 30-IV-1992, F. Gómez Vigide, GV 2915; ibidem, al oeste de la ciudad, 29TPJ1861, $430 \mathrm{~m}$, en orillas de caminos y arcenes, 30IV-1992, F. Gómez Vigide, GV2914; Mondoñedo, 29TPJ3310, en la orilla de praderas, 8-VIII-1973, F. Gómez Vigide, GV 2913; ibidem, 29TPJ21, 
en praderas, 8-VII-1973, GV 2912. OURENSE: Verín, 29TPG3145, 350 m, en pradera, 28-VII-1973, F. Gómez Vigide, GV 2915. PonTEvedra: Pontevedra, pr. Lourizán, 29TNG2795, 10 m, en pastos de orillas, 28-V-1974, F. Gómez Vigide, GV 2917; Vigo, pr. Samil, 29TNG1873, 10 m, en pastos de ribera, 16-VI-1973, F. Gómez Vigide, GV 2916; Tui, río Miño, 29TNG3055, 10 m, en riberas, 20-IV-1974, F. Gómez Vigide, GV 2918.

Novedad regional. Su areal se extiende hasta el Trásos-Montes portugués.

\section{Taraxacum fulgidum G. E. Haglund}

ESPAÑA: Lugo: Becerreá, Cruzúl, 29TPH5245, 430 m, en comunidades de Lauro-Quercetum ilicis subass. genistetosum falcatae sobre calizas, 24-V-1983, F. J. Silva-Pando 373, LOU 11006.

Afín a T. pinto-silvae Soest, diferenciándose por tener el lóbulo foliar terminal muy grande, hasta de 55,4$83,9 \times 46,4-58,3 \mathrm{~mm}$, triangular con los márgenes convexos, pecíolos frecuentemente alados, y las filarias de adpresas a reflejas y emarginadas. Novedad regional.

\section{Taraxacum gallaecicum Soest}

ESPAÑA: OURENSE: Rubiá, Escrita, entrada primer túnel, 29TPH7704, 600 m, en calizas, 30-IV-1988, F. J. Silva-Pando 4788, LOU 10248; Viana do Bolo, entre Solveira y el río Bibei, 29TPG5867, 750 m, en pastos de sombra, 12-IV-1997, F. Gómez Vigide, GV 2920.

Especie, que repartida por el $\mathrm{N}$ y E peninsular, solo era conocida en Galicia por su indicación locotípica (A Coruña: Santiago de Compostela) (Soest 1954: 140s). Especie afín a T. marginellum H. Lindb. Novedad provincial.

Taraxacum hispanicum $\mathrm{H}$. Lindb.

ESPAÑA: OURENSE: Rubiá, Pardollán, 29TPH70, $500 \mathrm{~m}$, en pastos, 12-V-1983, F. Gómez Vigide, GV 2921.

Novedad regional.

\section{Taraxacum lambinonii Soest}

ESPAÑA: LuGo: Becerreá, km 4 carretera de Becerreá a Navia de Suarna, 29TPH5448, 550 m, 25-III-1983, F. J. Silva-Pando 322 et cols., LOU 11102.

Otra especie afín a T. marginellum H. Lindb. y T. gallaecicum Soest, de la que se diferencia por los aquenios rosados, que crece en la mitad Norte de la Península Ibé- rica. Aunque es novedad regional, Bellot (1968: 140) menciona a "T. laevigatum (Willd.) DC." como característica de la Clase Festuco-Brometea en Galicia, aunque sin mencionar localidad concreta. T. laevigatum en realidad es un nombre que comprende numerosas microespecies de la sección Erythrosperma (Vašüt, 2003), por lo que la cita de Bellot pudiera tratarse de T. braun-blanquetii, T. gallaecicum o T. lambinonii.

\section{Taraxacum marklundii Palmgr.}

T. subhamatum M. P. Christ.

s/1.: "In regione littorea", B. Merino, LOU 1319/1 (sub. T. subhamatum M.P. Christ., Soest, 1954: 139). ESPAÑA: A CoRuÑa: PedrouZo, Arca, 29TNH5251, $268 \mathrm{~m}$, borde de camino junto a casas, 9-XII2006, A. Galán 1831, USP; Muros, Louro-San Francisco, 29TMH9334, $20 \mathrm{~m}$, en arcenes y orillas de caminos, 10-XII-2007, F. Gómez Vigide, GV 2922. Lugo: Lugo, Muralla Romana, 29TPJ16, 430 m, en grietas, 8-III-1992, F. Gómez Vigide, GV 2923. Pontevedra: Combarro, 29TNG2497, $6 \mathrm{~m}$, en el empedrado de una calle, 5-XII-2003, $\mathrm{A}$. $\mathrm{Ga}$ lán 366, USP; Cambados, 29TNH1506, $5 \mathrm{~m}$, base de un muro junto al paseo marítimo, 7-XII-2006, A. Galán 1832, 1833, 1834, USP.

Novedad para La Coruña y Pontevedra.

\section{Taraxacum nordstedtii Dahlst.}

\section{T. lusitanicum Soest}

ESPAÑA: Lugo: Lugo, Campiña de Muxa, 29TPJ1966, $430 \mathrm{~m}$, en prados encharcados, 26-IV-1991, F. Gómez Vigide, GV2924. OuRENSE: Rubiá, sobre Pardollán, 29TPH70, $500 \mathrm{~m}$, en pastizales de secano sobre sustrato calizo, 12-V-1983, F. Gómez Vigide, GV 2925. PoNtevedra: Vila de Cruces, pr. Asorei, 29TNH6834, c.600 m, 20-IV-1973, F. Gómez Vigide, GV 2927; Ponteareas, 29TNG4169, c.100 m, arcenes y pastos de orilla, 5-V-1963, F. Gómez Vigide, GV 2926.

Novedad provincial para Lugo y Pontevedra. La única cita regional corresponde al Monte Testeiro (Cerdeira, Irixo, Ourense) por Bellot (1950: s/p), en un prado subhúmedo. Se ha citado para Portugal de Trás-os-Montes (Soest 1948: 6), indicando que es una planta de praderas pantanosas en la Europa boreal.

\section{Taraxacum obovatum (Willd.) DC.}

T. obovatum subsp. ochrocarpum Soest - T. officinale Wigg. var. obovatum (DC.) Merino f. runcinatum Rouy.

ESPAÑA: OURENSE: Rubiá, cerro Peraliña, pr. Biobra, 29TPH7604, 700 m, sobre roquedo calizo, 6-V-1985, F. Gómez Vigi- 
de, GV 2929; ibidem, sobre el embalse de Peñarrubia, 29TPH7903, c. 460 m, en sustrato calizo, 3-IV-1984, F. Gómez Vigide, GV 2931; ibidem, sobre Pardollán, 29TPH7802, c. $800 \mathrm{~m}$, en pastos efímeros, 12-V1983, F. Gómez Vigide, GV 2930.

Especie muy variable. Citada por Merino (1906: 466, sub T. officinale var. obovatum), aunque plantea algunas dudas por carecer sus ejemplares de aquenios desarrollados; posteriormente lleva la planta a la variedad runcinatum (Merino 1916: 35). En ambos casos señala su abundancia en Galicia sin citar ninguna localidad. Laínz (1955: 139s) descartaba su presencia en Galicia, por lo menos en su forma típica, aunque menciona la posibilidad de que aparezcan en las comarcas sudorientales gallegas. Giménez Azcárate et al. (1991: 170, sub subsp. ochrocarpum) y Giménez \& Amigo (1996: 141 sub subsp. ochrocarpum) aportan dos localidades sobre sustratos calizos, como los nuestros.

\section{"Taraxacum palustre (Lyons) Symons"}

Fagúndez (2008: 156) citó está especie del municipio de Ferrol, pero una vez visto el pliego de respaldo (SANT 56434) descartamos que pertenezca a esta especie 0 a otra de la sección Palustria, pues las brácteas externas del involucro en la sección son más anchas y con un reborde blanquecino también bastante ancho. No podemos concretar la determinación por la falta de los aquenios.

\section{Taraxacum pinto-silvae Soest}

ESPAÑA: A CoRUÑa: Boimorto, Corredoiros, 29TNH7162, 491 m, 19-IV-1988, M. Romero, SANT 019658. Lugo: Cervantes, Penas de Aperdao, 29TPH7339, 1590 m, 5-VII-1986, F. J. Silva-Pando \& F. Pérez Caramés, LOU 13875; ibidem, Higón, km 17 carretera a Navia, 29TPH5952, 420 m, en repoblación, 28-IV-1988, F. J. Silva-Pando 4711, LOU 10210, 20559; Folgoso de Caurel, devesa del Pico Formigueiros, 29TPH5116, 1183 m, 6-V-2006, A. Galán 1898, USP; Caurel, de Moreda a La Devesa, bajo avellaneda, 29TPH5421, 790 m, 6-V-2006, A. Galán 1899, USP. OuREnSE: Serra do Invernadeiro, Vega de Meda, 29TPG6484, 1200 m, 17-IV-1973, S. Castroviejo, MA 198393; Bande, Porto Quintela, 29TNG8447, $560 \mathrm{~m}$, en pastizal bajo carballeira segada y antropizada (Arrhenatheretalia), 24-II-2005, I. Pulgar, SANT 52377.

Esta especie y T. nordstedtii son las especies de la sección Celtica más frecuentes en las montañas de la Península Ibérica, pero T. pinto-silvae pertenece a un grupo de poblaciones ibéricas con los lóbulos inferiores muy dentados y alargados que llegan a confundirse con el pecío10. Novedad regional, frecuente en todas las provincias españolas y portuguesas limítrofes con Galicia.
Taraxacum rubicundum (Dahlst.) Dahlst.

ESPAÑA: OURENSE: Viana do Bolo, entre Seoane y Hermida, cantera caliza supra Humoso, 29TPG5867, 900 m, calizas, 2-V-1992, F. J. Silva-Pando 7762, V. Rodríguez Gracia \& X. R. García, LOU 21028.

T. rubicundum se distingue por sus aquenios violáceos de pequeñas dimensiones, con costillas anchas casi lisas excepto en el ápice. Novedad regional.

\section{Taraxacum sundbergii Dahlst.}

ESPAÑA: Lugo: De Moreda a Devesa, 29TPH5321, 700 m, 6-V2006, A. Galán 1990, USP. OuRENSE: Humoso, in pratis et ericetis, B. Merino, LOU 1319/3.

La disposición asimétrica de los lóbulos foliares, unida al lóbulo terminal frecuentemente inciso, a los pecíolos alados y a las filarias externas a menudo purpúreas, diferencian esta especie del resto de las de la sección $R u$ deralia. Afín a ésta es T. maculosum A.J. Richards, de herbazales nitrófilos del norte del Lugo (junto a la iglesia de Meira, 29TPH3886, 498 m, s.f., P. Montserrat, JACA 200671). Novedad regional.

\section{Luzula congesta (Thuill.) Lej.}

L. multiflora subsp. congesta (Thuill.) K. Richt.- L. campestris var. pallens Merino, nom. illeg., non Asch.\& Graebn. [Typus: Prope Galdo (Lugo): Rodz. Franco] LOU 1642/1!.

ESPAÑA: PonTEvedRa: Baiona, baixo Monterreal, 29TNG1264, $10 \mathrm{~m}$, herbazais en cantil mariño, 2-V-2004, X. R. García Martínez 7604, GM 7604, det. S. Talavera 2010.

Según Montserrat (1964: 505) y Kirschner et al. (2002: 108), la planta descrita de Galdo (Lugo) por Merino (1904: 472) y posteriormente citada de Humoso y Penouta (Merino 1909: 70), corresponde a esta especie. El pliego que indicamos como representativo de la variedad, único existente en el herbario LOU, se ajusta bien a la descripción de la misma y entra dentro de la variabilidad de la especie, destacando las cabezuelas sentadas, salvo en algún caso, alguna pedunculada erecta, en sus tépalos externos largamente acuminados, los internos emarginado-escotados y aristados (Phyllis perigonii stramineis longe cuspidatis, Merino 1904: 472), los primeros de longitud mayor y todos de color castaño claro (perianthi phylla pallida, Merino 1909: 70). Según Kirschner et al. (1.c., mapa 90), se desarrolla en el Noroeste Ibérico, así como en otras localidades 
de la cornisa atlántica, desde el Sur de Portugal hasta Noruega e Islandia. En Fernández Piedra \& Talavera (2010: 193) encontramos una única mención para Galicia de esta juncácea y ahora presentamos el testimonio de referencia para la provincia indicada en esa obra. A destacar la altitud y la ecología en las que hemos encontrado la planta. En el Herbario LOU no hay pliegos de Humoso o Penouta. En cambio, la L. campestris variedad capitata Merino (Merino 1909: 70 [Typus: en terrenos feraces de Camposancos] Holotypus LOU 1642/6, indicado aquí) habrá de llevarse a L. campestris (L.) DC. in Lam. \& DC.

Sobre el género, recordar la cita de $L$. sudetica (Willd.) Schreb. (sub L. campestris DC. var. alpina? E. Meyer) de Merino (1909: 70s) que ya Montserrat (1.c.: 498) parecía aceptar. Hemos visto el pliego de referencia (LOU 1642/4) y corresponde a la especie, caracterizada entre otras, por las estrofíolos de 0,1 a $0,2 \mathrm{~mm}$. Fernández Piedra \& Talavera (2010: 194) solo la indican del Pirineo central, mientras que Kirschner et al. (2002: 209, mapa 89) la hace llegar hasta, aproximadamente, el País Vasco-Santander y habiendo además una cita de "Alto de la Farragosa, Somiedo, [29TQH36] de Mayor et al. (1978: tabla adjunta, inv. 28), además de un par de citas de Montserrat (1.c.: 498) para el Occidente de la Cordillera Cantábrica; por el contrario, Kirschner (1996: 6) sólo recoge localidades pirenaicas.

\section{Luzula lactea (Link) E. H. F. Meyer}

ESPAÑA: A Coruña: Zas, Coto do Muiño, Arco da Piosa-pr. Dolmen, 29TNH0266, 345 m, matorral sobre esquistos-pizarras, 2-VI2010, M.J. Rozados, F. J. Silva-Pando et al., LOU 36877. PonteveDRA: Vila de Cruces, noroeste del monte Garrio sobre Asorei, 29TNH6833, c. 650 m, 11-VII-1963, F. Gómez Vigide, GV 11021, 11022; Rodeiro, cumbres de la Sierra de O Faro, 29NH9019, c. 1100m, 25-VI-1992, F.Gómez Vigide, GV 11022..

Novedad provincial de una planta frecuente en los macizos montañosos gallegos del Este y Sur de Galicia aunque conocemos también la cita de la cumbre de Faro (Pontevedra) (Laínz 1971: 31). Destacamos la altitud a la que la hemos encontrado; Fernández Piedra \& Talavera (2010: 211) fijan un mínimo altitudinal de $600 \mathrm{~m}$ para la especie. Efectivamente en Galicia, conocíamos como cota más baja la publicada en Crespí et al. (1994: 18) de Campo Lameiro (Pontevedra) de $610 \mathrm{~m}$.

\section{Commelina communis L.}

ESPAÑA: A CoRUÑa: Muros, Louro, 29TMH9434, $20 \mathrm{~m}$, propagada en una huerta, 30-IX-2009, F. Gómez Vigide, GV 9086. PoNTE-
VEDRA: Tomiño, Barrantes, en islote en cauce del río Pego, 29TNG1951, 100 m, 4-IX-2008, J. B. Blanco-Dios, LOU 34986.

Citada por Pino et al. (2010: 395) de Ribadavia (Ourense) y por Romero Buján \& Amigo (2010: 111) de Tomiño (Pontevedra) y Ourense. Esta última localidad se basa en el pliego (SANT 54325) que mencionan Talavera et al. (2010: 118) de las orillas del río Miño en la ciudad de Orense. Señalan también Talavera et al. (1.c.) la presencia de Tradescantia pallida (Rose) D.R. Hunt del E y S de España, pero para Galicia ya vemos indicada su presencia en Rodríguez Gracia et al. (2008: 302) aunque como especie ornamental.

Ahora la encontramos en Muros (A Coruña) donde es novedad provincial. Ha de atenderse a su carácter expansivo, visto el ya conocido de T. fluminensis.

\section{Canna indica L.}

ESPAÑA: Lugo: Foz, Cangas de Foz, 29TPJ3431, $18 \mathrm{~m}$, en zanja húmeda, 27-VIII-2009, R. Pino \& J. J. Pino, LOU 34767.

Ya conocida de las otras provincias litorales de Galicia, donde cada vez es más abundante: González (1988: 25), Galán (2007: 268), Bernárdez (2006: 96) o Camaño et al. (2008: 11). Pino et al. (2010: 395) ampliaban su distribución a la provincia de Ourense. Ahora aparece en las costas de clima templado de Lugo donde es novedad provincial.

\section{Carex rostrata Stokes in With}

ESPAÑA: OURENSE: Carballeda, Peña Trevinca, lagoa de Mermiñeira, $1900 \mathrm{~m}$, bordes de la laguna, 21-VII-1987, F. J. Silva-Pando, E. Valdés Bermejo et al., LOU 09569; A Veiga, Xares, Lagoa do Oce10, 29TPG7577, $1650 \mathrm{~m}$, en roquedos pizarrosos, 14-VI-2008, F. J. Silva-Pando 14472, F. Gómez Vigide et al., LOU 35653; ibidem, A Ponte, pr. 0 Fial, 29TPG7677, $1800 \mathrm{~m}$, en borde de charca turbosa, 6-VIII-2009, F. J. Silva-Pando 15371, X. R. Garcia Martínez y F. Gómez Vigide, LOU 36830. ibidem, al sureste del Monte Tuirrieiro, 29TPG7978, c. 1800 m, 3-VIII-1984, F. Gómez Vigide, GV 9383; Manzaneda, laguna-turbera al sur de Cabeza de Manzaneda, 29TPG4279, 1387 m, 18-VII-1983, F. Gómez Vigide, GV 9384; Viana do Bolo, en la cumbre de Cabezo Grande, 29TPG6766, c.1400 m, 25-VII-1996, F. Gómez Vigide, GV 9385.

Laínz (1953: 161) conjeturaba su presencia en Galicia (sub C. ampullacea Good.), algo que Vicioso (1959: 162s) denotaba con la indicación "Galicia (Merino herb. ex Lainz)". Posteriormente, Laínz (1968a: 36) la herboriza en una laguna entre Ramilo y Pradorramisquedo [¿Laguna de Lama de Grúa?]. Rodríguez Gracia et al. (1989: 77) confirman su presencia en la provincia, en base a la primera lo- 
calidad, aunque añaden la de la Coruña, sin que realmente exista ninguna cita, tal como hemos comprobado en la base de datos que sirvió para elaborar el Catálogo Regional, del cual algunos de nosotros fuimos autores. Luceño (1994: 58, 2007: 162) no la señala en las provincias de A Coruña y Pontevedra. Tampoco aparece ninguna referencia para Galicia en Aedo et al. (1994: 91) a pesar de lo afirmado por Romero Buján (2008: 54). Es de destacar la baja altitud, c. $1400 \mathrm{~m}$, en que aparece en dos de las localidades, que contrasta con la que aparece en el resto del Noroeste ibérico. Y dentro de esta misma sección Vesiacariae se ha citado $C$. vesicaria L. de la isla de Ons en Souto \& de Sá (2005: 81). No hemos podido conseguir el pliego de respaldo y después de visitar el lugar y examinar las imágenes publicadas en Souto \& de Sá (1.c.), descartamos la cita, que se corresponde realmente con $C$. laevigata.

\section{Cyperus michelianus (L.) Link}

ESPAÑA: Pontevedra: Tui, Caldelas de Tui, reveros del río Miño, 29TNG3456, en arenales de ribera, 12-IX-1962, F. Gómez Vigide, GV 9427.

Castroviejo (2007: 23) omite la sigla 'Po' en la síntesis del género Cyperus, habiéndola ya señalado Merino (1909: 203) en la desembocadura del río Miño, cita que ahora reforzamos con la nuestra de Caldelas de Tui. Recientemente ha sido señalada para Ourense por Amigo \& Pulgar (2006: 49).

\section{Cyperus involucratus Rottb.}

ESPAÑA: Lugo: Xove, A Barxa, 29TPJ2239, $28 \mathrm{~m}$, en borde de camino, escasa, 27-VIII-2009, R. Pino \& F. Pardavila, LOU 34732.

Especie ornamental que se comporta como adventicia con suma facilidad, Fagúndez (2007a: 112). A las citas conocidas de Pontevedra (Blanco 1999: 31, Fagúndez 1.c., Pino et al. 2010: 396) y A Coruña (Fagúndez 1.c, 2008: 16, Pino et al. 1.c.), añadimos ahora la de Xove que es novedad para Lugo y no nos extranaría que se encontrara pronto en Ourense.

Eichhornia crassipes (Mart.) Solms. in A. DC.

ESPAÑA: PontevedRa: 0 Grove, Lagoa Bodeira, 29TNH0702, $3 \mathrm{~m}$, en laguna litoral de aguas dulces, 28-VII-2010, S. González \& M.J. Rozados, LOU 37195.
Planta cultivada en algunos estanques gallegos pero la encontramos naturalizada en esta laguna litoral. Fagúndez (2007), la recoge como posible invasora, pero señala que no se ha encontrado asilvestrada aún en Galicia. Las citas más cercanas corresponen a González Costales (2007: 190) de un lagunazo en la ría del Eo (Arnao, Castropol, Asturias) y Galán (2007: 322), que la indica como naturalizada en Douro Litoral en Portugal, habiéndose dectectado por primera vez en España de Alicante (1988). No obstante, véanse los comentarios de Pino et al. (2010: 395) sobre su naturalización en Punta Balea (Cangas, Pontevedra). Fernández et al. (2006: 72) ya la recogían en un inventario de la zona.

\section{Rhynchospora modesti-lucennoi Castrov.}

ESPAÑA: Pontevedra: Moaña, Domaio, San Lorenzo, 29TNG2583, 325 m, gándara de San Lorenzo, suelo turboso, 27-I-2010, D. Álvarez \& S. Rial, LOU 34963.

Feliz hallazgo de esta ciperácea que se creía extinguida en Galicia. Ortiz et al. (1998: 98) la incluyeron dentro de su listado de flora rara y amenazada de Galicia pero posteriormente Ortiz Herrera et al. (2004: 462) ya la señalaban como extinta en Pontevedra por la desecación de las Gándaras de Budiño al igual que Luceño \& Martín (2007: 102). Todos los catálogos de la región incluyen la provincia de A Coruña (Niño et al. 1994: 191, Rodríguez Gracia et al. 1989: 77 y Romero Buján 2008: 55) en base a la cita de Buch (1951: 75, sub. R. glauca Vahl) de Carnota, al pie del monte Pindo, donde no se ha vuelto a encontrar. Todas las citas gallegas de R. rugosa (Vahl) Gale han de llevarse a nuestra especie, a pesar de lo dicho por Romero Buján (1.c.) quien lo menciona como taxon independiente de $R$. modesti-lucennoi. En Galicia se encuentra incluida en el Catálogo gallego de especies amenazadas, en la categoría de "en peligro de extinción". Nuestra población, con mas de un centenar de ejemplares, se sitúa en una turbera de pequeña extensión y constituye de facto la población más numerosa de la Península Ibérica (Ortiz et al. 2004: 463). La exigua superficie que ocupa y los posibles usos forestales son los mayores peligros con los que se enfrenta. Urge el desarrollo de un plan de conservación y recuperación así como la protección integral de la turbera.

\section{Agropyrum cristatum subsp. pectinatum (M. Bieb.)} Tzvelev

ESPAÑA: Pontevedra: Lalín, Monte Carrio, 29TNH6832, c. $800 \mathrm{~m}$, orillas de pista de generadores eólicos, 19-VI-2009, F. Gómez 
Vigide, LOU 36826.

Se la ve, poco frecuente, en claros de matorral de Ulex europaeus y Erica umbellata. La localidad más cercana es Villafer (León) en el límite con Zamora (Alonso et al. (2003: 121). Recordemos aquí la cita palentina de Laínz (1968: 594, sub A. pectiniforme) ampliada en Aedo et al. (2001: 98). Novedad regional.

\section{Bromus inermis Leyss.}

ESPAÑA: Pontevedra: Lalín, Monte Carrio, 29TNH6832, c. $800 \mathrm{~m}$, en las orillas de pistas de generadores eólicos, 19-VI-2009, F. Gómez Vigide, LOU 36825.

Como la anterior, y más abundante que ella, crece en el matorral formado por Ulex y Erica. Probablemente fueron introducidas hace años como forraje del conejo silvestre. Está citada de León (Argüelles et al. 2004-2005: 173s), del Pais Vasco (Campos et al. 2002) y Soria (Segura et al. 1998a: 204, 1998b: 45), existiendo también varias citas de Salamanca y Valladolid [ANTHOS, 28-VIII-2009]. Novedad regional.

\section{Eleusine indica (L.) Gaertner}

ESPAÑA: A CoRuÑa: Noia, paseo marítimo, $5 \mathrm{~m}$, en rendijas, 1X-2010, F. Gómez Vigide, LOU 37197.

Citada ya de antiguo para España por Willkomm (1870: 46) de los suburbios de San Sebastián, no fue hasta Fagúndez (2007a: 112) que se citó de lugares antropizados en Santiago de Compostela. En la Península Ibérica se distribuye principalmente por el litoral mediterráneo, donde puede comportarse como invasora según Sanz Elorza et al. (2004b: 167). Sin embargo, su presencia en zonas más septentrionales y atlánticas suele ser casual y no se documentan episodios invasores.

\section{Eragrostis mexicana var. virescens (J. Presl.) S. D. Koch \& Sánchez Vega}

E. virescens J. Presl.

ESPAÑA: Pontevedra: Pontevedra, A Parda, pr. Guardería infantil, 29TNG3097, $50 \mathrm{~m}$, en lugares removidos, 11-VIII-2009, J. B. Blanco-Dios, LOU 37199.

De la Península Ibérica ha sido citada por Llamas et al. (2002: 126), para la provincia de León, y por Verloove
\& Sánchez Guillón (2008: 155), para Huelva. Los primeros autores incluyen algunas características de la planta. Novedad para la Flora Gallega.

\section{Paspalum notatum Flüggé}

ESPAÑA: A CoRuÑa: Noia, Paseo Marítimo, 29TNH03, $5 \mathrm{~m}$, en rendijas, 1-X-2010, F. Gómez Vigide, LOU 37197. Pontevedra: Cangas, Hío, Viñó, 29TNG1279, 40 m, márgenes de caminos localmente abundante, 8-II-2009, J. J. Pino \& R. Pino, LOU 34268; ibidem, Hío, Viñó, 29TNG1279, $40 \mathrm{~m}$, en bordes de cultivo, localmente abundante, 21-XI-2010, J. J. Pino, R. Pino \& A. Pino-Cancelas, LOU 34979. OURENSE: Ourense, río Miño, 29TNG9289, en céspedes y ribazos en la orilla del río, 8-VIII-2008, F. Gómez Vigide, GV 10435.

De Santiago de Compostela (A Coruña) lo citaba Fagúndez (2007a: 113) como novedad para Galicia. Posteriormente, se incluía sin ningún comentario en Pino et al. (2009: 120). Aunque Fagúndez (1.c.) advertía de su aparente escaso poder invasivo, nosotros consideramos que se trata de una gramínea a vigilar. Tiene un gran potencial como especie pionera en suelos degradados y, al tratarse de una planta apomíctica (Dahmer et al. 2008: 381, Witt et al. 2008: 236) su dispersión puede verse favorecida por la fijación de genotipos adaptados a determinados ambientes. En todo caso, es abundante en las zonas de transición de las dunas de la playa de Barra a los cultivos circundantes. Novedad para Ourense.

\section{Pennisetum clandestinum Huchst. ex Chiov.}

ESPAÑA: A CoRuÑa: Muros, Louro, en setos y al lado de muros, 30-IX-2010, F. Gómez Vigide, LOU 37198. Pontevedra: Bueu, Trasouto, 29TNG1784, $110 \mathrm{~m}$, en herbazales nitrófilos, 10-XII-2010, J. J. Pino \& R. Pino, LOU 34994; Cangas, San Roque, 29TNG1778, 40 m, en zonas ruderales, 25-XII-2010, R. Pino \& J. J. Pino, LOU 34995.

Planta originaría del Este de África, que está introducida en muchos países templados y tropicales. En España se conoce desde 1995 (Pyke 2008: 101) para el Levante español, encontrándose además en Valencia, Alicante, Sevilla, Huelva, Cádiz y las islas Canarias. Uno de los autores (A.G.de M.) la ha visto en Asturias en suelos arenosos muy removidos, pero no tenemos pliego de respaldo. Sólo hemos podido encontrar tres plantas con flores, mientras que mayoritariamente se reproduce vegetativamente, formando céspedes monoespecíficos, tal como señalan (Scholz et al. 2006: 72, Pyke 2008: 101), a veces muy difíciles de erradicar. Shouling \& Phillips (2006: 549) indican que es una especie muy inusual dentro del género, por su reduci- 
da inflorescencia, y las aristas deben buscarse dentro de las vainas foliares superiores. Novedad para la Flora gallega, región donde habrá que vigilar su expansión.

\section{¿Poa subcaerulea Sm.?}

P. pratensis subsp. irrigata (Lindm.) Tzvelev

Sobre la reciente cita de Fagúndez (2007b: 156) para la zona de Ferrol (A Coruña), donde nos ponían como "única referencia gallega"... "que los autores consideran dudosa", recordemos aquí las dudas planteadas por Laínz (1955: 148), Hernández Cardona (1978: 149) y Aedo et al. (1987: 453) sobre un pliego herborizado por Bellot en Carnota. Todos estos autores señalaban la proximidad de las plantas estudiadas, la mayoría de arenales costeros, con la nuestra; por el contrario, para el Pais Vasco (Aseginolaza et al. 1985: 971) y Navarra (Catalán \& Aizpuru 1986: 165) se aceptaba su presencia, aunque sin referirse a los autores citados anteriormente. Recientemente, Pyke (2003: 161) la cita para Barcelona como "introducida", señalando que diversas características de las hojas y tallo ayudan a separarla de $P$. pratensis. Planta a seguir en el futuro.

\section{Asphodelus fistulosus L.}

ESPAÑA: Pontevedra: Vigo, arcenes en Gándaras de Castrelos, 29TNG27, 17-III-2009, F. Gómez Vigide, GV 11172.

Después de las citas de Gómez Vigide et al. (2006: 65) para Montefurado, aparece ahora abundante en los arcenes de Gándaras de Castrelos. En Galicia, habrá de considerársele taxon alóctono aunque no invasor. No sería extraña su presencia futura en los arenales gallegos a tenor de lo dicho por Campos \& Herrera (2009: 248). Novedad provincial.

\section{Muscari neglectum Ten.}

ESPAÑA: Lug0: Quiroga, Montefurado, 29TPG4794, 315 m, en prados naturales, 14-III-2009, J. L. Camaño LOU 34309; ibidem, Montefurado, 29TPH4694, 300 m, en zonas sedimentarias del río Sil, escasa, 9-IV-2010, R. Pino, J. J. Pino, F. J. Silva-Pando \& A. Pino, LOU 34820.

Hasta 1985 no fue hallada esta pequeña liliácea en Galicia (Gómez Vigide 1985: 376) restringida al municipio de Rubiá (Giménez \& Amigo 1996: 100). Sin embargo nosotros la hemos observado en el vecino municipio de
Vilamartín de Valdeorras y ahora en los prados mediterráneos de los antiguos meandros del río Sil. Novedad para Lugo.

Ornithogalum bourgaeanum Jord. \& Fourr.

O. umbellatum L. auct. gal.

ESPAÑA: Lugo: Monforte de Lemos, Tor, Seoane, 29TPH11, en viñedo, 18-IV-1982, J. Amigo \& M. Romero, LOU 19435 sub O. umbellatum L.; ibidem, 29TPH1910, 290 m, IV-2008, en viñas, J. B. Blanco-Dios LOU 34988. Ourense: Rubiá, Sierra de Encina de la Lastra, sobre Biobra, 29TPH7606, 850-950 m, en caliza, 1-V-1987, F. J. Silva-Pando \& cols., pro parte sub 0 . umbellatum L.; ibidem, sobre Biobra (Serra da Encina da Lastra), 29TPH7506, 400 m, calías con exposición S, 1-V-1987, X. R. García Martínez 2383, U. García Aldao, V. Rodríguez Gracia, F. J. Silva Pando \& E. Valdés Bermejo, GM 2383; ibidem, Picos de Oulego, 29TPH7009, 800-860 m, calías, 12-VI-2009, X. R. García Martínez 8501, A. Guitián \& F. J. Silva Pando, GN 8501; Manzaneda, entre As Ermitas e Requias, prope Santipedre, 29TPG5284, 600 m, xistos, X. R. García Martínez 5202, V. Rodriguez Gracia \& F. J. Silva-Pando, GM 5202.

Desde que fue encontrada por primera vez en Galicia por Laínz (1967: 44) su estatus taxonómico no ha estado claro. Como O. umbellatum L. s.l. la vemos citada principalmente de las calizas de Rubiá (Laínz l.c., Rodríguez Gracia 1986: 23, Giménez \& Amigo 1996: 106), de Trigal (Carballeda, Ourense) por Ortiz \& Rodríguez (1987: 296) y de Castro de San Tomé (Ourense) por Rodríguez Gracia (1993: 251). De Lugo, sólo conocemos la referencia de San Clodio en Izco (1982: 154) procedente de un inventario. Martínez et al. [5-I-2011] reconocen bajo este binomen dos especies distintas: 0 . divergens Boreau y 0 . bourgaeanum, lo que obliga a revisar todos los pliegos para su asignación. Nuestras plantas, sin bulbillos secundarios y pedicelos florales inferiores erecto-patentes, se adscriben a 0 . bourgaeanum.

\section{Veratrum album $\mathrm{L}$.}

ESPAÑA: Pontevedra: Marín. Pastoriza, Pedra da Pousa, arroyo de Gorgadas, 29TNG2286, $380 \mathrm{~m}$, sobre el talud del arroyo, 21-III2009, D. Álvarez \& S. Rial, LOU 34369 (Figura 2).

Sorprendente hallazgo de esta liliácea en la vertiente norte de la Sierra del Jaján en un abedular degradado a una altura inferior a la mínima conocida hasta ahora de Galicia. Es planta relativamente frecuente en los macizos montañosos orientales de Galicia (Peña Trevinca, Courel y Ancares) y citada por primera vez por Colmeiro (1889: 160) 


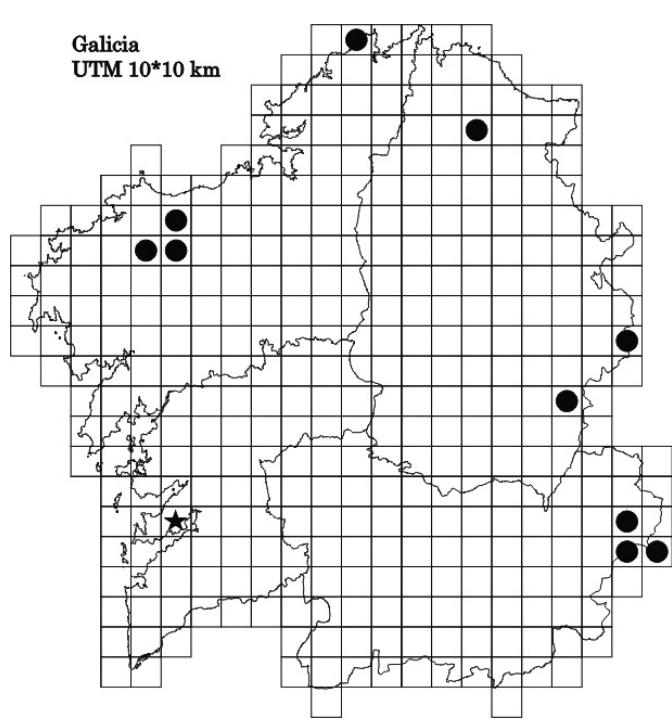

Fig. 2- Distribución de Veratrum album L. en Galicia.

en base al benedictino Sarmiento. Más al Norte, Merino (1909: 45) la encuentra en la cima del Cuadramón (Valadouro, Lugo), siempre a una altura superior a $10 \mathrm{~s} 1100 \mathrm{~m}$. Sin embargo, en Galicia también ha sido encontrada a una altura menor, tal vez en un ejemplo de pedinosis: en los ambientes montanos de la Serra de A Capelada (Cariño, A Coruña)(Perille et al. 2005: 270, Gómez Vigide et al. 2006: 65) y en torno a los municipios de Santa Comba, Val do Dubra, Coristanco y Carballo (Bellot \& Casaseca 1960: 238, Gómez Vigide et al. 1.c.), sobre afloramientos de carácter ultrabásico (Figura 6). Aparece ahora una población muy localizada pero de unos 300 ejemplares, si bien con muy pocas plantas florecidas. Se trata de un elemento relicto que debió ser mucho más abundante tras la finalización de la glaciación de Würm y que mantiene exiguas poblaciones en lugares apartados, al ser evitada por los grandes herbívoros por su elevada toxicidad (Kleijn \& Steinger 2002: 361). Novedad provincial.

\section{Chasmanthe floribunda (Salisb.) N.E. Brown}

Antholyza floribunda Salisb.

ESPAÑA: A CoRuÑa: Muros, Louro, 29TMH9334, 10 m, naturalizada, 13-11-2004, F. Gómez Vigide, GV 10862.

Novedad para la flora gallega. Planta de origen surafricano que Sánchez et al. (2009: 307) citan de Cádiz como novedad para la flora europea e indican que las citas de Almeida (1999), Sanz-Elorza \& al. (2004a) y Cortés (1994) de C. aethiopica (L.) N.E. Brown pueden corresponder a esta especie, con la que ha sido confundida a menudo. Sánchez Gullón \& Weickert (2004: 297) menciona C. aethiopica de Huelva. Nuestra planta tiene el tubo de color rojo asalmonado, con el tépalo superior más largo de color rosa claro a blanquecino, la parte proximal del tubo del periantio de $12-15 \times 1 \mathrm{~mm}$ y girada, y la parte distal mide 30 $\times 7 \mathrm{~mm}$, siendo la transición entre ambas gradual y no formando sacos, mientras que en la planta descrita por Curtis (1804: 561) y Glodblatt (2002: 403s) el diámetro de la parte proximal es de unos $3 \mathrm{~mm}$, de aproximadamente $1 / 3 \mathrm{del}$ diámetro de la parte distal de periantio.

\section{Iris foetidissima $\mathrm{L}$.}

ESPAÑA: OURENSE: O Barco de Valdeorras, Xagoaza, 29TPH6400, $480 \mathrm{~m}$, en zona sombría y húmeda, escasa, 20-VI-2009, J. J. Pino, R. Pino \& A. Pino, LOU 34511.

Aunque es una planta muy frecuente en el litoral, el primero en citarla de Galicia, Planellas (1852: 373) lo hizo curiosamente del interior: del Viso (Santiago de Compostela) y de los Nogales. La determinación parece confirmada por Pau (1924: 71) aunque no se ha vuelto a encontrar en dichos lugares. No obstante, Merino (1909: 145) aporta dos nuevas localidades del interior, no muy lejanas por cierto de la nuestra: "en parajes sombríos y muy húmedos de las parroquias de Saa y Diomondi (finca denominada las Cortes)". Rodríguez Guitián et al. (2000: 63) la mencionan de diferentes lugares de Lugo, como diferencial de la variante de Evonymus europaeus de la asociación Omphalodo nitidae-Coryletum avellanae Amigo, Giménez de Azcárate \& Romero 1994, subas. coryletosum avellanae, comunidad donde la hemos encontrado nosotros. Novedad para Ourense.

Iris planifolia (Mill.) Fiori \& Paol.

ESPAÑA: OURENSE: Rubiá, Biobra, Sierra da Encina da Lastra, As Galegas, A Tara, 29TPH7208, 1112 m, sobre roquedos calizos, 27VI-2009, F. J. Silva-Pando, R. Pino, J. J. Pino et al., LOU 34551.

Interesantísima localidad, muy alejada de las del centro y sur de la Península Ibérica, siendo las provincias más cercanas Beira Litoral en Portugal (Morales Abad 1991: 260) y Cáceres en España (Morales Abad \& Fernández Casas 1990: 99s). Existe una cita de Díaz-González (1981) para Asturias "Peña Rueda, Quiros", pero no hemos encontrado ningún pliego de respaldo en los Herbarios OVI 
y LEB [GBIF, 30-VI-2009]. Planta de floración invernal de la que sólo encontramos las típicas hojas y cápsulas del fruto. Novedad regional.

Watsonia bulbillifera Mathews \& Bolus.

ESPAÑA: A CoRuña: Muros, Louro, 29TMH9334, $10 \mathrm{~m}$, en ribazos y cunetas, 19-IV-2008, F. Gómez Vigide, GV 10863. PoNTEVEDRA: Ponteareas, As Cachadas, cerca de Cenedo, 29TNG4170, en pastos al lado del camino, 30-V-1973, F. Gómez Vigile, GV 10864.

Iridácea de origen surafricano, que se desarrolla en bordes de camino. Citada por Ramos Lopes \& Pinto da Silva (1980: 7) de diversas partes de Portugal; en el Norte portugües se cultivaba como ornamental y se escapa de cultivo. Las flores son color rojo brillante, interiormente más pálido, tubo del periantio de hasta $4,5 \mathrm{~cm}$ y lóbulos de hasta $2,5 \mathrm{~cm}$, oblongos los externos y obovados los internos, mientras que presenta bulbillos en los nudos del tallo (Campbell 1986: 384, Goldblatt 2002: 402). En Galicia florece a finales de la primavera. Novedad para la Flora española.

\section{Hedychium gardnerianum Sheppard ex Ker-Gawler}

ESPAÑA: Lugo: Viveiro, En el acceso a la playa de Abrela, 29TPJ1137, 50 m, en talud con Rubus sp., 27-VIII-2009. R. Pino \& F. Pardavila LOU 34734.
Citada por Silva-Pando et al. (2010: 24) de Muros (A Coruña) aparece ahora naturalizada en un talud sobre el litoral. Especie a vigilar por sus tendencias expansivas para evitar futuros peligros. Recordemos que se trata de una especie incluida dentro de las 100 más peligrosas del planeta por su capacidad invasora (Lowe et al. 2000: 4). La hemos visto en floración hasta diciembre. Novedad para Lugo.

\section{Passiflora caerulea L.}

eSPAÑA: Pontevedra: Cangas, Darbo, Sierra Poniente, 29TNG1678, 49 m, como trepadora sobre Quercus robur, 27-IX-2009, R. Pino \& J. J. Pino, LOU 34772; ibidem, Darbo, Alto da Portela, 29TNG1782, 155 m, 27-IX-2009, R. Pino \& J. J. Pino LOU 34777.

Alonso (1820: 265) y Blanco-Dios (1999: 51) la mencionan de A Coruña y Pontevedra como cultivada, pero Rodríguez Romero et al. (2008: 340) ya la señalan como naturalizada en Ourense. En las zonas en que la hemos herborizado es subespontánea.

\section{AGRADECIMIENTOS}

A Tomás Emilio Díaz González, Carlos Aedo y los responsables de los herbarios JACA, LEB, MA, MACB, SANT, SEV-Históricos e Instituto Sánchez Cantón de Pontevedra por las informaciones y facilidades para la consulta de los herbarios. A Feliciano Gómez Vigide O.F.M., Alberto Guitián, Adrián Pino-Cancelas y Antonio Prunell Tuduri por su compañía y estímulo en algunas excursiones.

\section{BIBLIOGRAFÍA}

Aedo, C.; Herrá, C.; Laínz, M; Loriente, E.; Morteno Moral, G \& Patallo, J. 1985. Contribuciones al conocimiento de la flora montañesa, IV. Anales Jard. Bot. Madrid 42(1): 197-213.

Aedo, C.; Aldasoro, J. J.; Argüelles, J. M.; Díaz Alonso, J. L.; Díez Riol, A.; González del Valle, J. M.; Laínz, M.; Morteno Moral, G \& Patallo, J. \& Sánchez Pedraja, Ó. 1994. Contribuciones al conocimiento de la flora cantábrica, II. Fontqueria 40: 67-100.

Aedo, C.; Aldasoro, J. J.; Argüelles, J. M.; Carlón, J. L.; Díez Riol, A.; González del Valle, J. M.; Laínz, M.; Morteno Moral, G \& Patallo, J. \& Sánchez Pedraja, Ó. 2000. Contribuciones al conocimiento de la flora cantábrica, IV. Bol. Cien. Nat. R.I.D.E.A. 46: 7-119.

Aedo, C.; Aldasoro, J. J.; Argüelles, J. M.; Carlón, J. L.; Díez Riol, A.; Gómez Casares, G.; González del Valle, J. M.; Guillén Oterino, A.; Laínz, M.; Morteno Moral, G \& Patallo, J. \& Sánchez Pedraja, Ó. 2001. Contribucio- nes al conocimiento de la flora cantábrica, V. Bol. Cien. Nat. R.I.D.E.A. 47: 7-52.

Aedo, C.; Herrá, C.; Loriente, E. \& Morteno Moral, G. 1987. Contribuciones al conocimiento de la flora montañesa. Anales Jard. Bot. Madrid 44(2): 445-457.

ALmeIDA, J. D. 1999. Flora exótica subespontánea de Portugal continental (Plantas vasculares). Universidad de Coimbra. Coimbra.

ALONSO LóPEZ, J. 1820. Consideraciones generales sobre varios puntos históricos, políticos y económicos a favor de la libertad y fomento de los pueblos. II. Geología, Climatología y Botánica de Ferrol y su comarca. Imp. Repulles. Madrid.

Alonso Redondo, R.; De Paz Canuria, E.; García González, M. E.; Puente Garçía, E. \& Penas Merino, A. 2003. Notas corológicas sobre la flora vascular de la prvincia de León. Stud. Bot. 21: 121-126.

Amaral Franco, J. Do. 1986. Juniperus L. En: S. Castroviejo, M. Laínz, G. López González, P. Montserrat, F. Muñoz Gar- 
mendia, J. Paiva \& L. Villar (Eds.), Flora iberica 1: 181188. Real Jardín Botánico, CSIC. Madrid.

Amigo VÁzquez, J. 1984. Estudio de los matorrales y bosques de la Sierra del Caurel (Lugo). Tesis doctoral. Universidad de Santiago de Compostela. Santiago de Compostela.

Amigo, J. \& Pulgar, I. 2006. Apuntes sobre la flora gallega, XVII. Stud. Bot. 24: 45-54.

AnthoS. 2010-2011. Sistema de información de las plantas de España. Real Jardín Botánico, CSIC Fundación Biodiversidad. Recurso electrónico en www.anthos.es.

Argüelles, J.M.; Carlón, L.; Gómez Casares, G.; González del Valle, J. M.; Laínz, M.; Moreno Moral, G. \& SÁNChez Pedraja, Ó. 2004-2005. Contribuciones al conocimiento de la flora cantábrica, VII. Bol. Cien. Nat. R.I.D.E.A. 49: 147-194.

Aseginolaza, C.; Gómez, D.; Lizaur, X.; Montserrat, G.; Morante, G.; Salaverría, M. R.; Uribe-Echeverría, P. M. \& Alejandre, J. A. 1985. Catálogo florístico de Álava, Vizcaya y Guipúzcoa. Gobierno Vasco. Victoria.

Bañares, Á.; Blanca, G.; Güemes, J.; Moreno, J. C. \&. Ortíz, S. (Eds.) 2004. Atlas y Libro Rojo de la Flora Vascular Amenazada de España. Dirección General para la Biodiversidad, Publicaciones del O.P.A.N. Madrid.

Bellot, F. 1947. Revisión crítica de las especies del género "Hippocrepis"de la Península e Islas Baleares. Anales Jard. Bot. Madrid 7: 197-334.

Bellot, F. 1950. Mantissa Stirpium Gallaeciae. Trab. Jard. Bot. Santiago 1: 0-0.

Bellot, F. 1968. La vegetación de Galicia. Anales Inst. Bot. Cavanilles 24: 3-306.

Bellot, F. \& CASASECA, B. 1960. Adiciones y correcciones a la flora de Galicia. Anales Inst. Bot. Cavanilles. 17(1): 233-248.

Benedí, C. 1997. Chamaesyce Gray. En: S. Castroviejo, C. Aedo, C. Benedí, M. Laínz, F. Muñoz Garmendia, G. Nieto Feliner \& J. Paiva (Eds.), Flora iberica 8: 286-297. Real Jardín Botánico, CSIC. Madrid.

Benito Alonso, J. L. (Ed.). 1995. Noticia de la primera campaña de la AHIM (Ancares-Caurel, Junio de 1994). Boletín de la Asociación de Herbarios Ibero-Macaronésicos. 0: 9-12.

Bernárdez Villegas, J.G. 2006. Estudio florístico de la 'Illa de Ons', parque nacional maritimo-terrestre de las islas Atlánticas de Galicia. Organismo autónomo Parques Nacionales. Ministerio de Medio Ambiente. Madrid.

Blanco-Dios, J. B. 1999. Catálogo de la Flora Vascular. Parque Natural Monte Aloia. Consellería de Medio Ambiente. Xunta de Galicia. Santiago de Compostela.

Brummitt, R. K. 1997. Two subspecies of Calystegia silvatica (Kit.) Griseb. (Convolvulaceae) in the mediterranean region. Lagascalia 18: 338-340.

Buch, H. 1951. Uber die Flora und Vegetation Nordwest Spaniens. Commentiationes Biologicae. Societas Scientiarum Fennica. Helsinki 10(17):1-98

Camaño Portela, J. L.; Silva-Pando, F. J.; Pino Pérez, J. J. \& Pino PÉrez, R. 2008. Asientos corológicos LOU, 2005. Boletín BIGA. 4: 5-21.
Campbell, E. 1986. Watsonia Miller. En: S. M. Walters et al. (Eds.), The European Garden Flora 1: 384-385. Cambridge University Press. Cambridge.

Campos, J. A.; Berastegui, A.\& Darquistade, A. 2002. Sobre algunas plantas poco conocidas del País Vasco y zonas limítrofes. Est. Mus. Cienc. Nat. Álava 17: 125-130.

Campos, J. A. \& Herrera, M. 2009. Diagnosis de la Flora alóctona invasora de la CAPV. Dirección de Biodiversidad y Participación Ambiental. Departamento de Medio Ambiente y Ordenación del Territorio. Gobierno Vasco. Bilbao.

Carbo Nadal, R.; Mayor Lopez, M.; Andrés Rodríguez, J. y LOSA QuinTANA, J. M. 1977. Aportaciones al catálogo florístico de la provincia de León. 11. Acta Bol. Malacitana 3: 63-120.

Carlón, L.; González del valle, J. M.; Laínz, M.; Moreno Moral, G.; Rodríguez Berdasco, 0. \& SÁnchez PedraJA, 0. 2010. Contribuciones al conocimiento de la flora cantábrica, VIII. Documentos del Jardín Botánico Atlántico (Gijón) 7: 1-95.

Carrasco, M. A.; García-Camacho, R. \& Martín-Blanco, C. J. 2005. Notas de flora hispánica, V. Bot. Complut. 29: 27-29.

Castro, M.; Freire, L. \& Prunell, A. 1989. Guía das árbores de Galicia. Ed. Xerais. Vigo.

Castroviejo, S. 1972. Flora y cartografía de la vegetación de la Península de Morrazo (Pontevedra). Tesis doctoral (inéd.). Universidad Complutense de Madrid, Madrid.

Castroviejo, S. 2007. Cyperus L. En: S. Castroviejo (Coord.), Flora Iberica 18: 8-27. Real Jardín Botánico, C.S.I.C. Madrid.

Castroviejo, S \& Lago, E. 1990. Una Linaria (Scrophulariaceae) de la costa gallega, mal interpretada. Nova Acta Científica Compostelana (Bioloxía). 1: 5-10.

Castroviejo, S. \& Velayos, M. 1995. Sedum L. ser. Rupestria Berger (Crassulaceae) en la Península Ibérica. Anales Jard. Bot. Madrid 53(1): 145-146.

Castroviejo, S. \& Velayos, M. 1995. Sedum L. En: S. Castroviejo, C. Aedo, M. Laínz, R. Morales, F. Muñoz Garmendia, G. Nieto Feliner \& J. Paiva (Eds.), Flora iberica 5: 121153. Real Jardín Botánico, C.S.I.C. Madrid.

Catalán, P. \& Aizpuru, I. 1986. Datos florísticos de las cuencas de los ríos Bidasoa y Urumea. Munibe 38: 163-16.

CAVANILLES, A. J. 1791. Icones et descriptiones plantarum quae aut sponte in Hispania crescunt, aut in hortis hospitantur. Vol. I. Madrid.

Chater, A.O. \& Walters, S.M. 1976. Senecio L. En: T. G. Tutin, V. H. Heywood, N. A. Burges, D. M. Moore, D. H. Valentine, S. M. Walters \& D. A. Webb (Eds.), Flora Europaea 4: 191-205. Cambridge University Press. Cambridge.

Colmeiro y Penido, M. 1889. Enumeración y revisión de las plantas de la Peninsula Hispano-Lusitana é islas Baleares, con la distribución geográfica de las especies, y sus nombres vulgares, tanto nacionales como provinciales, V (Monocotiledóneas y criptogamas). Madrid.

Cook, C. D. K. 1986. Ranunculus (subgen. Batrachium) L. En: S. Castroviejo, M. Laínz, G. López González, P. Montserrat, F. Muñoz Garmendia, J. Paiva \& L. Villar (Eds.), 
Flora iberica 1: 285-298. Real Jardín Botánico, C.S.I.C. Madrid.

CORTÉs, J. 1994. The exotic flora of Gibraltar. Almoraima 11: 155-169.

Costa Tenorio, M.; Morla Juaristi, C. \& Saínz Ollero, H. 1984. Notas fitocorológicas del interior peninsular. Collect. Bot. (Barcelona) 15: 167-172.

Crespí, A. L.; Portela, J.; GonzÁlez Hernández, M. P.; Rodríguez Gracia, V.; Castro cerceda, M.; Freire García, L.; Prunell Tuduri, A.; Gamarra Mondelo, E.; Astor Camino, J.; Martín Fragueiro, I.; Iglesias Garrote J.L.; Castroviejo Bolibar, S.; Medina Trigo, R.; Sanmartín Bienzobas. L. A.; Toimil Trigo, M. J.; Silva-Pando. F. J.; García Martínez, X. R.; Valdés-Bermejo, E. \& Gómez vigide, F. 1994. Flora del Noroeste de la Península Ibérica. Exssicata-fascículo 5, n 351-450. Centro de Investigaciones Forestales. CIFA-Lourizán. Pontevedra.

CurTis, W. 1804. Antholyza aethopica. Bot. Mag. 15-16: 561.

Dahmer, N.; Schifino-Wittmann, M. T.; Dall'Agnol, M. \& CAstro, B. 2008. Cytogenetic data for Paspalum notatum Flügge accessions. Sci. Agric. (Piracicaba, Braz.) 65(4): 381-388.

DíAz GonZÁlez, T. E. 1981. Enciclopedia Temática de Asturias. Botánica. Gijón.

FAGÚNDEZ, J. 2007. Nuevos datos de flora vascular exótica en Galicia (NO de la Península Ibérica). Lazaroa. 28: 111-114.

FAGÚNDEZ, J. 2007. Estudio de la flora vascular del concello del Ferrol (A Coruña, NO de la Penísnula Ibérica). Citas florísticas. Nova Acta Ci. Compostelana (Bioloxía) 16: 155-159.

FAGÚNDEZ, J. 2008. Estudio de la flora vascular del concello de Ferrol (A Coruña, NO de la Península Ibérica). Flora exótica. Nova Acta Ci. Compostelana (Bioloxía) 16: 11-17.

FERNÁNDEZ ARECES, M. P. 1989. Flora y Vegetación Rupicola de la Cordillera Cantábrica, Montes de León y Cuenca Alta del río Ebro. Revisión Taxonómica del Género Saxifraga $L$. Sección Dactyloides Tausch en el Norte de la Península Ibérica. Tesis doctoral. Universidad de León. León.

Fernández Areces, M. P.; PÉrez CARro, F. J. \& Díaz-GonzÁLEZ, T. E. 1987. Estudio del Cheilanthion hispanicae Rivas Goday 1955 cm. Saénz de Rivas \& Rivas-Martínez 1979 y comunidades afines, en el Sector Orensano-Sanabriense (Provincia Carpetano-Iberico-Leonesa). Lazaroa 7 : 207-220.

Fernández Cordeiro, A.; Álvarez Graña, D.; Dasairas BouZada, A.; Rial Pousa S.; Pastoriza Barreiro, A. \& PiñeiRo Lemos, J. 2006. O espazo natural de Punta Balea. Grupo de Anelamento Anduriña. Cangas.

Fernández Piedra, M. P. \& Talavera, S. 2010. Luzula DC. En: S. Talavera, M. J. Gallego, C. Romero Zarco \& A. Herrero (Eds.), Flora iberica 17: 187-224.

FröHner, S.E. 1998. Alchemilla L. En: F. Muñoz Garmendia \& C. Navarro (Eds.), Flora iberica 6: 195-357. Real Jardín Botánico, CSIC. Madrid.

Galán, A. 2007. Canna L. En: S. Castroviejo, M. Luceño, A. Galán, P. Jiménez Mejías, F. Cabezas \& L. Medina (Eds.),
Flora iberica 18: 268-270. Real Jardín Botánico, C.S.I.C. Madrid.

Galán, A. 2007. Eichhornia Kunth. En: S. Castroviejo (coord.), Flora Iberica 18: 321-322. Real Jardín Botánico, C.S.I.C. Madrid.

Galán, A. 2010. Taraxacum. En: S. Castroviejo (coord.), Flora Iberica 16 (www.floraiberica.org). Real Jardín Botánico, C.S.I.C. Madrid.

García Martínez, X. R.; Valdés-Bermejo, E.; Silva-Pando, F. J.; Rodríguez Gracia, V. \& Gómez Vigide, F. 1991. Aportaciones a la flora de Galicia, IV. Nova Acta Ci. Compostelana (Biol.) 2: 41-56.

GBIF. 2010-2011. Global Biodiversity Information Facility in Spain. Nodo Nacional de Información sobre Biodiversidad. www.gbif.es.

Gimenez de AzcÁrate Cornide, J.; Romero, M. I. \& Amigo, J. 1991. Apuntes sobre la flora gallega, XI. Bol. Soc. Brot. Serie 2. 64: 159-172.

Gimenez de AzCÁRate Cornide, J. \& Amigo VÁzquez, J. 1996. Inventario da flora vascular de afloramentos calios de $\mathrm{Ga}$ licia. Caderno da Área de Ciencias Biolóxicas (Inventarios) XII. Publicacións do Seminario de Estudos Galegos. Edicións do Castro. Sada (A Coruña).

GlodBatT, P. 2002. Chasmanthe N.E. Brown. En: Flora of North America Editorial Committee (Eds.), Flora de North America North of Mexico 26: 403-404. Oxford University Press. New York \& Oxford.

GlodBatt, P. 2002. Watsonia Miller. En: Flora of North America Editorial Committee (Eds.), Flora de North America North of Mexico 26: 401-402. Oxford University Press. New York \& Oxford.

Gómez Vigide, F. 1985. Algunas aportaciones al conocimiento de la flora gallega. Anales Jard. Bot. Madrid 41(2): 367-380.

Gómez Vigide, F.; García Martínez, X. R.; Valdés-Bermejo, E.; Silva-Pando, F. J. \& RodrígueZ Gracia, V. 1989. Aportaciones a la flora de Galicia, III. En: F. J. Silva-Pando (Ed.), Sobre Flora y Vegetación de Galicia: 101-121. Consellería de Agricultura. Santiago de Compostela.

Gómez Vigide, F.; García Martínez, X. R.; Pino Pérez, R.; GonzÁlez Domínguez, J.; Blanco-Dios, J. B.; Camaño PorteLa, J. L.; Pino Pérez, J. J.; Silva-Pando, F. J. \& VÁzQuez Míguez, A. C. 2006. Aportaciones a la Flora de Galicia. VII. Nova Acta Ci. Compostelana (Bioloxía) 14: 57-68.

Gómez Vigide, F.; García Martínez, X. R.; Silva-Pando, F. J.; GonzÁlez Domínguez, J.; Blanco-Dios, J. B.; RodrígueZ GonzÁlez, A.; Rial Pousa, S.; Álvarez Graña, D.; CAmaño Portela, J. L.; Pino Pérez, J. J. \& Pino Pérez, R. 2007. Aportaciones a la flora de Galicia, VIII. Nova Acta Ci. Compostelana (Bioloxía). 15: 53-63.

GonZÁLEZ, E. 1988. Flora alóctona gallega I. Universidad de Santiago de Compostela.

GonzÁlez Costales, J. A. 2007. Plantas alóctonas invasoras en el principado de Asturias. Consejería de Medio Ambiente, Ordenación del Territorio e Infraestructuras. Gobierno del Principado de Asturias. Oviedo. 
Güemes, J. 2009. Antirrhinum L. En: C. Benedí, E. Rico, J. Güemes \& A. Herrero (Eds.), Flora iberica 13: 134-166.

Guitián, J.; Amigo, J.; Ortiz, S. \& RodríGueZ-OubiñA, J. 1985. Apuntes sobre la flora gallega III. Trab. Comp. Biol. 12: 197-203.

Hergueta, A. R., Gamallo, G. L. \& Orduña, J. 1999. Aportaciones a la flora de los Montes Aquilianos (Noroeste de la Península Ibérica). Nova Acta Ci. Compostelana (Bioloxia) 9: 159-164.

Hernández Cardona, A. M. 1978. Estudio monográfico de los géneros Poa y Bellardiochloa en la Península Ibérica e islas Baleares. Dissertationes Botanicae 46: 1-365.

IzCo, J. 1982. Linario amethysteae-Calenduletum, asociación de los viñedos mediterráneos gallegos. Anales Real. Acad. Farmacia 48: 147-160.

IzCo, J.; Guitián, J.; Amigo, J. \& RodríGueZ-OubiÑA, J. 1985b. Apuntes sobre la flora gallega, 2. Trab. Comp. Biol. 11: 131-140.

KirSChNER, J. 1996. Luzula sect. Luzula (Juncaeae) in Spain. Pl. Syst. Evol. 200: 1-11.

KIRSCHNER, J. et al. 2002. Juncaceae 1: Rostkovia to Luzula, Species Plantarum: Flora of the World Part 6: 1-237. Australian Biological Resources Study. Canberra.

Kleijn, D. \& Steinger, T. 2002. Contrasting effects of grazing and hay cutting on the spatial and genetic population structure of Veratrum album, an unpalatable, long-lived, clonal plant species. Journal of Ecology 90: 360-370.

LAínZ, M. 1953. Adiciones inéditas del P. Baltasar Merino a su Flora de Galicia. Broteria Ci. Nat. 22(49): 155-168.

LAínZ, M. 1955. Aportaciones al conocimiento de la flora gallega. Broteria, Ci. Nat. 24: 108-143.

LAínZ, M. 1956. Aportaciones al conocimiento de la flora gallega, II. Anales Inst. Bot. Cavanilles. 14(0): 529-554.

LAínZ, M. 1967. Aportaciones al conocimiento de la flora gallega, V. Anales Inst. Forest. Invest. 12(0): 1-51.

LAínz, M. 1968. Aportaciones al conocimiento de la flora gallega, VI. M. Pub. Inst. Forestal Inv. Agr. 1-39 pp. Ministerio de Agricultura. Madrid.

LAínz, M. 1971. Aportaciones al conocimiento de la flora gallega, VII. I.F.I.E.: 1-38. Instituto Forestal de Investigaciones y Experiencias. Madrid.

LAÍnZ, M. 1974. Aportaciones al conocimiento de la flora gallega, VIII. Publ. INIA: 1-26.

LANGE, J. 1865. Pugillus plantarum imprimis hispanicarum, quas in itinere 1851-52 legit. IV. Vidd. Meddel. Dansk. Naturh. Foren. 1865: 225-339.

Llamas, F.; Acedo, C. \& Lence, C. 2002. De Plantis Legionensibus XVII. Lazaroa 23: 125-127.

Lowe, S.; Browne, M.; Boudjelas, S. \& De Poorter, M. 2000. 100 de las especies exóticas invasoras más dañinas del mundo. Aliens 12: 3-11.

LuCEÑo, M. 1994. Monografía del género Carex en la Península Ibérica e Islas Baleares. Ruizia 14: 1-140.

LuCEÑo, M. 2007. Carex L. En: S. Castroviejo (coord.), Flora Iberica XVIII: 109-250. Real Jardín Botánico, C.S.I.C. Madrid.

LuceÑo, M. \& MarTín, J. 2007. Rhynchospora Vahl. En: S. Castroviejo et al. (Eds.), Flora Iberica 17: 99-102. Real Jardín Botánico, CSIC. Madrid.
Martínez Azorin, M.; Crespo, M. B. \& Juan, A. 2011. Ornithogalum L. En: S. Castroviejo et al. (Eds.), Flora iberica 20, http://www.floraiberica.es, [5-I-2011].

Mayor, M.; Díaz-GonzÁlez, T. E.; FernÁndez Prieto, J. A. \& Lastra, J. J. 1978. Estudio ecológico del cervuno (Nardus stricta L.) en la Cordillera Cantábrica. Pastos 8(2): 183-194.

Merino, B. 1897. Contribución a la flora de Galicia. La vegetación espontánea y la temperatura en la cuenca del Miño. Tipografía Galaica, Tuy.

Merino, B. 1901. Contribución a la flora de Galicia. Suplemento III. Anales Soc. Esp. Hist. Nat. ser. II, 10: 167-199.

Merino, B. 1902. Viajes de herborización por Galicia. Razón y fé II: 82-89. (Hay edición facsimilar más reciente: B. MERINO, 1987: Viajes de herborización por Galicia. Seminario de Estudos Galegos. Santiago.)

Merino, B. 1905-1909. Flora Descriptiva e Ilustrada de Galicia. I, II, III. Tipografía Galaica. Santiago de Compostela.

Merino, B. 1912. Adiciones a la Flora de Galicia (al Tomo I). Broteria, ser. Bot. X: 125-140, 173-191.

Merino, B. 1916. Adiciones a la Flora de Galicia. Brotería, ser. Bot. XIV: 158-171.

Montserrat Recorder, P. 1964. El género Luzula en España. Anales Inst. Bot. Cavanilles 21(2): 407-541.

Morales, R. 1993. Clypeola L. En: S. Castroviejo et al. (Eds.), Flora iberica 4: 200-203. Real Jardín Botánico, CSIC. Madrid.

Morales Abad, M. J. 1991. Mapa 398 (adiciones). Iris planifolia (Miller) Fiori \& Paol. 18. Fontqueria XXXI: 260-261.

Morales Abad, M. J. \& Fernández Casas, J. 1990. Mapa 398. Iris planifolia Miller) Fiori \& Paol. 16. Fontqueria XXVII: 99-100.

Nieto Feliner, G. 1985. Estudio crítico de la flora orófila del Suroeste de León: Montes Aquilianos, Siera del Teleno y Sierra de la Cabrera. Ruizia 2: 5-239.

Niño Ricoi, E \& Losada Cortiñas, E \& Castro GonzÁlez, J. 1994. Catálogo da Flora Vascular Galega. Consellería de Agricultura, Gandería e Montes. Santiago de Compostela.

Ortega Olivencia, A. \& Devesa, J. A. 2007. Asperula L. En: J. A. Devesa, R. Gonzalo \& A. Herrero (Eds.), Flora iberica 15: 36-56 Real Jardín Botánico, CSIC. Madrid.

Ortiz, S. \& RodríGuez OubiÑA, J. 1987. Apuntes sobre la flora gallega, VI. Lazaroa 10: 295-298.

Ortiz, S.; Rodríguez OubiÑa, J. \& Pulgar, I. 1998. Unha primeira aproximación ao listado da flora rara e ameazada de Galicia (NO da Península Ibérica). Nova Acta Científica Compostelana (Bioloxía). 8:95-101.

Ortiz Herrera, M. A.; De Vega durán, C.; Berjano Pérez, R.; Sánchez Gullón, E. \& Talavera Lozano, S. 2004. Rhynchospora modesti-lucennoi Castrov. En: A. Bañares, G. Blanca, J. Güemes, J. C. Moreno \& S. Ortiz (Eds.), Atlas y Libro Rojo de la Flora Vascular Amenazada de España: 462-463. Dirección General para la Biodiversidad, Publicaciones del O.A.P.N. Madrid.

PaU, C. 1912a. Visita botánica al Desierto de la Palmas. Bol. Soc. Aragonesa Cienc. Nat. XI: 163-169.

PAU, C. 1912b. Plantas nuevas de la provincia de Madrid. Bol. Soc. Arag. Cienc. Nat. 11: 39-42. 
PAU, C. 1916. Notas sueltas sobre la flora matritense. Bol. Soc. Arag. Cienc. Nat XV: 158-172.

PAU, C. 1922. El herbario Planellas. Broteria, ser. Bot. 20(3): 120-129.

PAU, C. 1924. El herbario Planellas. Broteria, ser. Bot. 21: 43-82.

Paunero, E. 1952. Las especies españolas del género Alopecurus. Anales Inst. Bot. Cavanilles 10(2): 301-345.

Perille, M.; Pimentel, M.; Romero, D. \& Sahuquillo, E. 2005. Novedades de interés para la flora de Galicia (N.O. Península Ibérica). Nova Acta Ci. Compostelana (Bioloxía) 11: 269-271.

Plaza arregui, L \& Rodríguez HiRaldo, C. 2009. La recuperación de Linaria lamarckii. Conservación Vegetal 13: 16-17.

Pino Pérez, J. J.; Camaño, J. L. \& Pino Pérez, R. 2007. Asientos corológicos, LOU 2004. Boletín BIGA. 2: 40-120.

Pino Pérez, J. J.; Rial Pousa. D.; Álvarez Graña, S.; SilvaPando, F. J.; Camaño Portela, J. L. \& Pino Pérez, R. 2010. Contribución a la flora alóctona de Galicia. Bol. Auriense. 38-39: 385-402.

Pino Pérez, J. J.; Silva-Pando, F. J.; Camaño Portela, J. L. \& PINo PÉreZ, R. 2008. Asientos corológicos LOU, 2006. Bol. BIGA. 4: 23-36.

Pino Pérez, R.; Camaño Portela, J. L.; Pino Pérez, J. J. \& SilVA-PANDO, F. J. 2009. Asientos corológicos LOU, 2009. Bol. BIGA 6: 109-124.

Piñerio Portela, R.; Silva-Pando, F. J. \& Pino, R. 2007. Asientos para un atlas corológico de la flora occidental. Boletín BIGA 2: 133-148.

Planellas GiRalt, J. 1852. Ensayo de una flora fanerogámica gallega ampliada con indicaciones acerca de los usos medicos que se describen. Imprenta y Litografía de D. Juan Rey Romero, Santiago de Compostela.

Pujadas Salva, A. J. \& Plaza, L. 2010. Verbena L. En: S. Castroviejo (coord.), Flora Iberica 12: 13-21. Real Jardín Botánico, CSIC. Madrid.

Pyke, S. 2008. Contribución al conocimiento de la flora alóctona catalana. Collect. Bot. 27: 95-104.

Ramos Lopes, M. H. \& Pinto DA Silva, A. R. 1980. Plantas novas e novas áreas para a Flora de Portugal. XIII. Agron. Lusit. 40(1): 5-40.

Rigueiro Rodríguez, A. \& Silva-Pando, F. J. 1984. Aportaciones a la flora de Galicia, I. Anales Jard. Bot. Madrid 40 (2): 385-395.

Rivas Mateo, M. 1899. Datos para la flora gallega. Actas $R$. Soc. Esp. Hist. Nat. 28(99): 95-103.

RodríGUEz Gracia, V. 1983. Comentarios a la flora de Galicia, I. Bol. Auriense 13: 15-22.

RodríGUez Gracia, V. 1986. Comentarios a la Flora de Galicia. II. Bol. Auriense XVI: 31-38.

Rodríguez Gracia, V. 1993. Comentarios a la flora de Galicia, VI. Bol. Auriense 23: 249-254.

Rodríguez Gracia, V.; De Jesús GonzÁlez, J. A. \& Rodríguez Romero, R. A. 2008. Flora ornamental auriense. I. Duen de Bux, S.L. Ourense.
Rodríguez Gracia, V.; Gómez Vigide, F.; Valdés bermejo, E.; García Martínez, X. R. \& Silva-Pando, F.J. 1989. Catá$\log _{0}$ de la flora vascular gallega, I. Pterydophyta, Gymnospermae y Monocotiledoneas. En: F. J. Silva-Pando (Ed.), Sobre Flora y Vegetación de Galicia: 71-89. Consellería de Agricultura. Santiago de Compostela.

Rodríguez Gracia, V. \& Rodríguez Romero, X. 1999. Comentarios a la flora de Galicia, IX. Bol. Auriense XXIX: 177-181.

Rodríguez Guitián, M. A.; Amigo, J. \& Romero Franco, R. 2000. Aportaciones sobre la interpretación, ecología y distribución de los bosques supratemplados naviano ancarenses. Lazaroa 21: 51-71.

Rodríguez Romero, R. A.; De Jesús GonzÁlez, J. A.; RodríGUEz Romero, X.; Doce Fernández, M. \& Rodríguez GrACIA, V. 2008. Comentarios a la flora de Galicia, XII. Bol. Auriense. XXXVI: 337-344.

Romero Buján, M. I. 2007. Flora exótica de Galicia (noroeste ibérico). Bot. Complut. 31: 113-125.

Romero Buján, M. I. 2008. Catálogo da flora de Galicia. Universidade de Santiago de Compostela. Monografías do Ibader 1. Lugo.

Romero Buján, M. I. \& Amigo, J. 1993. Apuntes sobre la flora gallega. XII. Bol. Soc. Brot., 2a sér. 65: 195-203.

Romero Buján, M. I. \& Amigo, J. 2010. Una nueva Commelinaceae invasora en Galicia. Nova Acta Ci. Compostelana (Bioloxía) 18: 111-113.

Romero Zarco, C. 1999. Pisum L. En: S. Talavera, C. Aedo, S. Castroviejo, C. Romero Zarco, L. Sáez, F. J. Salgueiro \& M. Velayos (Eds.), Flora iberica 7(1): 482-486. Real Jardín Botánico, CSIC, Madrid.

SÁEZ, L. \& Bernal, M. 2009. Linaria Mill. S. Castroviejo (coord.), Flora Iberica 12: 232-324. Real Jardín Botánico, CSIC. Madrid.

SÁnchez Gullón, E. \& Weickert, P. 2004. A new species of Iridaceae for SW of Spain. Contribuciones a la flora vascular de Andalucía (España) (96-100). Acta Bot. Malac. 29: 297-298.

SÁnchez, I.; García-DE-Lomas, J. \& DANA, E. 2009. Aportaciones al conocimiento de la xenoflora gaditana. Lagascalia 20: 296-313.

Sanz Elorza, M.; Dana SÁnchez, E. D. \& Sobrino VesperiNAS, E. (Eds.). 2004. Atlas de las Plantas Alóctonas Invasoras en España: 146-147. Dirección General para la Biodiversidad. Madrid.

Sanz Elorza, M.; Dana Sánchez, E. D. \& Sobrino VesperiNAS, E. (Eds.). 2004. Eleusine indica (L.) Gaertner En: Atlas de las plantas alóctonas invasoras en España: 166-167. Dirección General para la Biodiversidad. Madrid.

Scholz, S.; Reyes-Betancort, J. A. \& Wilpret de la Torre, W. 2006. Notas corológico-taxonómicas de la Flora Macaronésica ( $\mathrm{N}^{0}$ 128-142). Bot. Macaronésica 26: 65-76.

Segura, A., Mateo, G. \& Benito, J. L. 1998a. Catálogo florístico de la provincia de Soria. Monografías Fl. Montib. 4.

Segura, A., Mateo, G. \& Benito, J. L. 1998b. De flora soriana, X. Fl. Montib. 8: 44-49. 
Shouling, C. \& Phillips, S. M. 2006. Pennisetum Richard. En: W. Zhengyi \& P. H. Raven (Eds.), Flora of China 22: 548552. Science Press. Beijing.

Silva-Pando, F. J. 1994. Flora y Series de Vegetación de la Sierra de Ancares. Fontqueria 40: 233-388.

Silva-Pando, F. J. 2008. Las plantas endémicas y subendémicas de Galicia. Bol. BIGA. 3: 9-150.

Silva-Pando, F. J.; Gómez Vigide, F.; García martínez, X. R. \& Blanco-Dios, J. B. 2000. Aportacións á flora de Galicia, VI. Nova Acta Ci. Compostelana, Biol. 10:21-33.

Silva-Pando, F. J.; Pino Pérez, R.; Pino Pérez, J. J.; García Martínez, X. R.; Morla Juaristi, C.; Cebolla Lozano, C.; Gómez Vigide, F.; Camaño Portela, J. L.; Rial PouSa, S.; Álvarez Graña, D.; Blanco-Dios, J. B. \& Paz RoSALES, M. 2010. Aportaciones a la flora de Galicia, IX. Nova Acta Ci. Compostelana, Biol. 18: 37-63.

Silva-Pando, F. J.; Rodríguez Gracia, V.; García Martínez, X. R. \& VAldÉS BeRMeJo, E. 1987. Aportaciones a la flora de Galicia, II. Bol. Soc. Brot., sér. 2a , 60: 29-68.

Soest, J. L. van. 1948. Sur quelques Taraxaca et Hieracia du Portugal. Agron. Lusit. X(I): 6-23.

SoEst, J. L. van. 1954. Sur quelque Taraxaca de Galice. Broteria Ci. Nat. 23(50) 11-111: 139-142.

Souto FigueroA, M. G. \& De SÁ Otero, M. P. 2005. Flora da Illa de Ons. Diputación Provincial de Pontevedra. Pontevedra.

Talavera, S.; Gallego, M. J. \& Herrero, A. (Eds.). 2010. Commelinaceae. En: S. Talavera, M. J. Gallego, C. Romero Zarco \& A. Herrero (Eds.), Flora iberica 17: 118-119. Real Jardín Botánico, CSIC. Madrid.

Talavera, S. \& GibBs, P. E. 1999. Teline Medik. En: S. Talavera, C. Aedo, S. Castroviejo, C. Romero Zarco, L. Sáez, F. J. Salgueiro \& M. Velayos (Eds.), Flora iberica 7(1): 141147. Real Jardín Botánico, CSIC. Madrid.

TEXIDOR y Cos, J. 1869. Apuntes para la Flora de España, ó Lista de plantas no citadas y raras en Galicia, partido judicial de Valladolid, provincia de Madrid y Cataluña. Revista de los progresos de las ciencias exactas, fisicas y naturales 18, n. ${ }^{\circ}$ 9: [597]-659.

Uribe-EcheVAría, P. M. \& Zorrakin, I. 2004. Claves ilustradas de Flora del Moncayo. Gobierno de Aragón. Zaragoza.
Valdés, B. 2010. Echium L. En: S. Castroviejo (coord.), Flora Iberica 11 (www.floraiberica.org). Real Jardín Botánico, C.S.I.C. Madrid.

VALDÉs , B.; OCAÑA, M. E. \& PARRA. 2000. Linaria lamarckii Rouy. En: G. Blanca et al. (Eds). Libro Rojo de la flora silvestre amenazada de Andalucía 2: 216-218.

Valdés, B.; Talavera, S. \& Fernández Galiano, E. 1987. Flora de Andalucía occidental. Ketres Editores, S.A. Barcelona.

Valdés Bermejo, E.; Silva Pando, F. J.; Rodríguez Gracia, V.; Gómez Vigide, F. \& García Martínez, X. R. 1995. Aportaciones a la flora de Galicia, V. Nova Acta Ci. Compostelana, Biol. 5: 135-152.

VAš̈UT, R. J. 2003. Taraxacum sect. Erythrosperma in Moravia (Czech Republic): taxonomic notes and the distribution of previously described species. Preslia 75: 311-338.

Verloove, F. \& SÁnchez Gullón, E. 2008. New records of interesting xenophytes in the Iberian Peninsula. Acta Bot. Malac. 33: 147-167.

Vicioso, C. 1959. Estudio monográfico sobre el género "Carex” en España. Instituto Forestal de Investigaciones y Experiencias. Madrid.

Walters, S. M. 1968. Alchemilla L. En: T. G. Tutin, V. H. Heywood, N. A. Burges, D. M. Moore, D. H. Valentine, S. M. Walters \& D. A. Webb (Eds.), Flora Europaea 2: 48-64. Cambridge University Press. Cambridge.

Willkomm, M. 1870. Senecio L. En: M. Willkomm \& J. Lange, Prodromus Florae Hispanicae 2: 111-125d. Sumtibus E. Schweizerbart (E. Koch). Stuttgart.

Willkomm, M. 1893. Suplementam Prodromi Florae Hispanicae. Stuttgart.

Willkomm, M. 1870. Eleusine Gärtn. En: M. Willkomm \& J. Lange, Prodromus Florae Hispanicae 1: 46. Sumtibus E. Schweizerbart (E. Koch). Stuttgart.

Willkomm, M. \& Lange, J. 1880. Prodromus Florae Hispanicae 3. Stuttgart.

Witt Cidade, F.; Miguel Dall’Ágnol, M.; Bered, F. \& TeiXeira de Souza-Chies, T. 2008. Genetic diversity of the complex Paspalum notatum Flügge (Paniceae: Panicoideae). Genet Resour. Crop Evol. 55: 235-246. 